\title{
La Dinámica de la Distribución del Ingreso ante Diferentes Políticas Monetarias
}

\author{
Raúl Oscar Sánchez ${ }^{1}$ \\ Universidad Nacional de La Plata \\ Maestría en Economía \\ Tutor: Daniel Heymann
}

25 de abril de 2008

1email: r_o_sanchez20030yahoo.com.ar 


\section{Resumen}

Este trabajo explora las implicancias distributivas así como los efectos en las variables macroeconómicas agregadas de diferentes políticas monetarias en una economía abierta habitada por agentes heterogéneos, sujetos a shocks agregados (tanto nacional como extranjero) e individuales. A efectos de llevar a cabo este trabajo, el modelo del agente representativo se extiende a uno compuesto por diferentes agentes con riesgos individuales no asegurables, que difieren en la productividad laboral. A tal efecto, se analiza una economía pequeña bajo un modelo de equilibrio general dinámico estocástico, donde se amplia el modelo Neo-Keynesiano propuesto por Galí y Monacelli [18], el cual se resolverá empleando el Toolkit desarrollado por Uhlig [7]. De este modo, la economía abierta será analizada para un contexto que combina las ideas Neo-Keynesianas de mercados incompletos y rigideces de precios con los "shocks tecnológicos positivos" de la teoría del Ciclo Económico Real. Se observan diferente conclusiones dependiendo de la políticas monetaria adoptada (objetivo de inflación doméstica, de inflación de precios $I P C$ y de fijación de carácter $P E G$ ). Asimismo, los diferentes tipos de shocks presentan resultados muy distintos entre sí, dependiendo de la política monetaria adoptada. 


\section{Índice general}

1. Introducción $r$

2. Literatura $\quad 4$

2.1. El Modelo Neo-Keynesiano . . . . . . . . . . . . . . . . . . . . . 6

3. El Modelo con agentes heterogéneos $\quad 8$

3.1. Las Familias . . . . . . . . . . . . . . . . . . . . . . 8

3.2. Inflación, Tasa de Cambio Real y los términos de intercambio . . . . . . . . 9

3.3. Las Firmas . . . . . . . . . . . . . . . . . . . . . . . 10

3.4. Algunas Identidades . . . . . . . . . . . . . . . . . . . 11

3.5. Riesgo Internacional Compartido . . . . . . . . . . . . . . . . . . . 12

3.6. El Equilibrio . . . . . . . . . . . . . . . . . . . . . . 13

3.6.1. Consumo mundial y su producción . . . . . . . . . . . . . . 13

3.6.2. Consumo y Producción en la Economía Pequeña . . . . . . . . . . . . . . 13

3.7. La Oferta: Dinámica Inflacionaria, Costos Marginales y la Brecha de Producción 14

3.8. Dinámica de Equilibrio . . . . . . . . . . . . . . . . . . . . . . 15

3.9. Políticas Monetarias Alternativas . . . . . . . . . . . . . . . 15

3.9.1. Economía Mundial . . . . . . . . . . . . . . . . . . . 15

3.9.2. La Economía Abierta . . . . . . . . . . . . . . . 16

3.10. Dinámica Distributiva . . . . . . . . . . . . . . . . . . . . . . . . 18

$\begin{array}{lr}\text { 4. Resultados } & 20\end{array}$

4.1. Comportamiento Agregado de la Economía . . . . . . . . . . . . . . . . . 21

4.1.1. Shock de Productividad Doméstica Agregada . . . . . . . . . . . . . 21

4.1.2. Shock de Productividad Externo . . . . . . . . . . . . . . . 26

4.2. Dinámica Distributiva . . . . . . . . . . . . . . . . . . . . . . . . . . . . . . . . . . . . . . .

4.2.1. Shock Individual . . . . . . . . . . . . . . . . . . . 32

5. Conclusiones 36

$\begin{array}{lr}\text { Bibliografía } & 39\end{array}$

A. Comprobaciones Matemáticas $\quad 1$

A.1. Fijación de precios óptimos en el modelo de Calvo . . . . . . . . . . . . . . . 1

A.2. Maximización de Utilidad . . . . . . . . . . . . . . . . . . . 2 


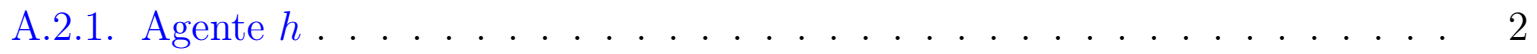

A.2.2. Análisis . . . . . . . . . . . . . . . . . . 3

A.2.3. Log-Linealización . . . . . . . . . . . . . . . . . . . . . . . 4

A.3. Costos Marginales . . . . . . . . . . . . . . . . . . . . . 4

A.3.1. Derivación de las ecuaciones IS . . . . . . . . . . . . . . . 6

A.3.2. Objetivo $C P I \ldots \ldots \ldots 7$

B. Solución de la Dinámica mediante el método de los coeficientes indetermina$\begin{array}{lr}\text { dos } & 8\end{array}$

B.1. Revisión del Toolkit . . . . . . . . . . . . . . . . . . . 8

B.1.1. Solución de la Matriz de Ecuación Cuadrática . . . . . . . . . . . . . . 10

B.1.2. Implementado las ecuaciones en el Toolkit . . . . . . . . . . . . . . 11 


\section{Índice de figuras}

3.1. Curva de Lorenz. . . . . . . . . . . . . . . . . . . . . . . . . . . . . 18

4.1. Funciones de Reacción (Modelo OID) . . . . . . . . . . . . . . . . . 23

4.2. Funciones de Reacción (Modelo OI-IPC) . . . . . . . . . . . . . . . . 24

4.3. Funciones de Reacción (Modelo OIF) . . . . . . . . . . . . . . . . 25

4.4. Situación de los Consumidores. Shock Agregado Nacional. . . . . . . . . 27

4.5. Situación de los Consumidores. Shock Agregado Externo. . . . . . . . . 29

4.6. Curva de Lorenz (2 Consumidores) . . . . . . . . . . . . . . . . . . 30

4.7. Funciones de Reacción del Coeficiente de Gini con 2 consumidores. . . 31

4.8. Curva de Lorenz (5 Consumidores) . . . . . . . . . . . . . . . . . . . . 32

4.9. Funciones de Reacción del Coeficiente de Gini con 5 consumidores. . . 32

4.10. Análisis del Coeficiente de Gini ante shock Individual (2 consumidores). 33

4.11. Análisis del Coeficiente de Gini ante shock Individual (5 consumidores) 34

4.12. Consumo Individual (5 consumidores) . . . . . . . . . . . . . 35 


\section{Índice de cuadros}

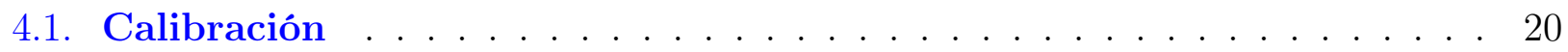

4.2. Relaciones (Shock Doméstico Agregado) . . . . . . . . . . . . . . . 31

4.3. Relaciones (Shock Internacional Agregado) . . . . . . . . . . . . . . 31 


\section{Capítulo 1}

\section{Introducción}

La macroeconomía moderna basa gran parte de su análisis a través de muchos microfundamentos, entre los que se pueden citar el horizonte infinito, los mercados competitivos, retornos constantes a escala y el agente representativo. Como bien explican Heer y Maußner [8], este ultimo supuesto se basa en el calculo intertemporal de los agentes homogéneos que buscan maximizar su utilidad a lo largo de su vida, quienes además actúan racionalmente. Sin embargo, con el correr del tiempo, la teoría comienza a evolucionar y los supuestos empiezan a levantarse, tal es así, por ejemplo, que el horizonte infinito es reemplazado por los modelos de generaciones superpuestas, o se abandonan los mercados perfectos por los modelos de competencia monopolística.

Sin embargo, el supuesto del agente homogéneo casi nunca es descartado, excepto en pocos trabajos. El empleo de agentes heterogéneos, tiene la ventaja de poder utilizarse en la teoría de la distribución, en la de los precios de activos o en las finanzas públicas. Esto es así, dado que los agentes pueden diferir en muchas características, como ser sus habilidades, educación, edad, número de hijos, posesión de activos, etc.

Otro de los supuestos tradicionalmente empleados en la literatura corresponde al de la economía cerrada, lo cual resulta impensable en el mundo real. En la economía moderna, los países interactúan con sus vecinos, y en esta era de globalización, las interacciones tienden a crecer exponencialmente. Dado que estos vínculos resultan más y más importantes, surgen muchas cuestiones a tener en cuenta en los modelos económicos. La consideración de una economía abierta conlleva nuevos desarrollos y muchos retos. Tradicionalmente, el análisis consiste en dos economías que interactúan. A veces, la interacción es mutua, otras, una economía pequeña es influenciada por otra mayor. En ambos casos, los economistas deben lidiar con un doble vector de variables macroeconómicas. Sin embargo, esos modelos no suelen considerar los tipos de cambio nominal y real, o los términos de intercambio.

Bajo este contexto, en el presente trabajo, se desarrolla un modelo en el que la política monetaria de la economía mundial resulta óptima, y la economía pequeña desarrolla políticas alternativas, en el que se explora cuales son las implicancias distributivas de cada política ante diferentes shocks (agregado e individual). Por tal motivo, haciendo uso del modelo propuesto por Galí y Monacelli [18] (que resulta una ampliación del tradicional de Equilibrio General 
Dinámico Estocástico), se modifica para contener agentes heterogéneos que están sujetos a shocks agregados e individuales que afectan su productividad así como sus canastas de consumo.

Asimismo, se consideran rigideces de precios con competencia imperfecta, lo cual resulta una de las principales consideraciones de la teoría Neo-Keynesiana.

Los diferentes agentes tratarán de maximizar su utilidad sujeta a una determinada restricción presupuestaria. La función de producción emplea trabajo como input, lo cual es provisto por las familias que difieren en su productividad. Asimismo, esta estará sujeta a shock agregados (doméstico y extranjero) e individuales.

En este trabajo se intentará analizar los siguientes puntos:

- ¿Cuáles son los efectos distribucionales de los shocks agregados e individuales?

- ¿Cómo afecta la política monetaria elegida?

- ¿Cómo influye la economía mundial?

A efectos de cumplir el objetivo, el modelo implementará el Toolkit desarrollafdo por Uhlig $[7]^{2}$, el cual resulta apropiado para analizar modelos de Equilibrio General Dinámico Estocástico. Básicamente, el procedimiento consiste en: obtener las condiciones de primer orden, establecer las ecuaciones de steady-state, log-linealizar las ecuaciones y calcular las funciones de reacción así como las simulaciones via el Toolkit. Tras encontrar el comportamiento dinámico de las variables, se analiza la dinámica de la desigualdad. Siguiendo a Cowell [3], nos concentramos en el coeficiente de Gini por dos rezones: en primer lugar, resulta ser una expresión muy sencilla además de ser un resumen de la curva de Lorenz, en segundo lugar, resulta fácil de implementar en el Toolkit.

En la Sección 2 de este trabajo se hace una revisión a la literatura que estudia tanto la teoría del Equilibrio General Dinámico, los Agentes Heterogéneos y la distribución del ingreso. La Sección 3 efectúa el planteo del modelo. La Sección 4 presenta una discusión de los resultados. Por último, en la Sección 5 se presentan las conclusiones del presente trabajo.

\footnotetext{
${ }^{2}$ Para más información acerca del Toolkit, ver http://www2.wiwi.hu-berlin.de/institute/wpol/html/toolkit.htm
} 


\section{Capítulo 2}

\section{Literatura}

En esta sección del trabajo, se hará una revisión de la literatura que emplea diferentes variantes del modelo Neoclásico de crecimiento o Neo-Keynesiano con agentes heterogéneos. Además, explicaré brevemente las principales características de una Economía Abierta NeoKeynesiana.

Siguiendo a Krusell y Smith [10], el supuesto del agente representativo no parece implicar un tratamiento serio de los microfundamentos. Sin embargo, ellos consideran que este supuesto tiende a ser razonable bajo dos circunstancias. La primera, se presenta cuando los supuestos empleados coinciden con los datos utilizados. La segunda posibilidad, en tanto (la cual es la explorada por los autores) es aquella en la que las variables agregadas del modelo teórico con una descripción más realista del contexto se comporta como el del agente representativo. El objetivo del trabajo es el de analizar si el modelo del agente representativo constituye una estrategia razonable. El principal resultado de este trabajo es que en el equilibrio estacionario, el comportamiento de los agregados macroeconómicos se puede describir como una función de dos estadísticos: la media de la distribución de la riqueza y el shock de productividad agregado.

Caselli y Ventura [19] estudian varias fuentes de heterogeneidad para el consumidor en un modelo de crecimiento y desarrollan herramientas para estudiar la evolución de la distribución del consumo, activos e ingresos. Ellos demuestran que el modelo de crecimiento con agente representativo puede generar una dinámica del consumo, riqueza e ingresos. Consideran un modelo con tres fuentes de heterogeneidad: riqueza inicial, habilidades no adquiribles y deseos de consumo. A pesar de esta heterogeneidad, el modelo admite un consumidor representativo y consecuentemente, exhibe una dinámica agregada que resulta similar a los modelos estándares de consumidor homogéneo. Examinan el comportamiento del consumo relativo del consumidor, riqueza e ingresos, y derivan ecuaciones cross-section que demuestran que estas cantidades están relacionadas tanto con las variables promedios como con las individuales.

Existen modelos en que los ingresos de los agentes son determinados determinísticamente, las familias difieren en su posesión de activos y la riqueza es indeterminada. Tal es el caso del trabajo de Chattarjee [2], que establece que si todos los agentes son similares y no sufren incertidumbre, el modelo colapsa a uno del agente representativo. El autor encuentra que la distribución inicial de la riqueza en una economía con agentes que viven infinitamente, que 
poseen preferencias homotéticas y la función de producción es neoclásica, permanece constante en el equilibrio sin interesar si esta se encuentra o no en steady state.

Otros trabajos recientes que analizan la dinámica de la distribución del ingreso son los de Banerjee y Newman [9], Ríos-Rull [6], y Krusell y Smith [11].

Banarjee y Newman [9] muestran que la distribución inicial de la riqueza puede tener implicancias cuantitativas importantes en el largo plazo. Ellos suponen otra fuente de equilibrio dentro de la elección de ocupación de los agentes. Estudian una dinámica dual del salario y la distribución del ingreso en el que la tasa de interés está dado exógenamente. En su modelo un salario bajo reduce la acumulación por los agentes pobres y de esta manera impide una oferta importante de trabajo. El modelo contiene cuatro tipos de actividades: idle (depositan dinero), contratados, auto-empleados y emprendedores (que organizan la producción y monitorean a los contratados pero no pueden controlar más de cierto número de trabajadores).

Castañeda, Díaz-Giménez y Ríos-Rull [14] estudian la dinámica de la distribución del ingreso en un contexto neoclásico con shocks de carácter individual, mercados incompletos y restricciones en el mercado de préstamos. Ellos presentan cuatro tipos de heterogeneidad del modelo de crecimiento neoclásico. El modelo analizado contiene las siguientes características: en primer lugar, incluyen dos factores de producción, trabajo y capital, y consecuentemente los agentes tienen dos fuentes de ingreso (salario y renta). En segundo lugar, los agentes están sujetos a un proceso estocástico exógeno en sus oportunidades de empleo, y trabajan si tienen la oportunidad de hacerlo. Se supone que este proceso no puede asegurarse. Otra característica es que los precios resultan endógenos. Esta implicancia y el supuesto de mercados competitivos determinan que tanto los ingresos del trabajo como los de capital son endógenos en el modelo. De este trabajo surgen tres resultados importantes. Específicamente, encuentran que la separación de la población en cinco grupos por tipos de empleo resulta suficiente para analizar la dinámica de la distribución del ingreso en los Estados Unidos. El rol que juegan los factores mobiles es insignificante en el modelo. Y la dinámica de la distribución del ingreso resulta independiente de la concentración de la riqueza.

Otro aspecto tradicionalmente tratado en la literatura de agentes heterogéneos corresponde a la importancia de la heterogeneidad de la riqueza debido a fluctuaciones económicas. Por ejemplo, se puede citar el trabajo de Banerjee y Newman [9] y Galor y Zeira [15] entre otros. En una economía calibrada del tipo Bewley, sin embargo, Krusell y Smith [10] muestran que la heterogeneidad de la riqueza no tiene impacto en la dinámica agregada. Adoptando un especificación Constant Absolute Risk Aversion-CARA (Aversión Absoluta al Riesgo Constante) consiguen determinar los precios y las demás variables agregadas macroeconómicas independientemente de la distribución de la riqueza. Más aún, el trabajo destaca que los mercados incompletos pueden tener implicancias importantes para la acumulación de capital, crecimiento de mediano plazo y ciclos económicos, incluso cuando la heterogeneidad de la riqueza no influye en las variables agregadas.

Por su parte, tradicionalmente la escasa literatura que trata la desigualdad y la política monetaria lo hace desde un enfoque empírico. El trabajo de Guiso, Haliassos y Japelli [5] indica 
que los pobres tienden retener más su dinero, en tanto que los ricos diversifican más. Por lo tanto, ante una inflación se ven perjudicados en mayor medida los del primer grupo. Los trabajos de Dolmas, Huffman y Wynne [4] y de Albanesi [1] destacan como las cuestiones de carácter politico afectan la inflación, estableciendo la política monetaria como función de la desigualdad.

Habiendo explorado los principales trabajos que analizan los ciclos económicos reales y la distribución del ingreso, podemos observar una de las principales contribuciones de este trabajo: se analiza una economía abierta en el que los agentes difieren en su productividad laboral, estando sujetos a shocks de carácter agregado e individual, en el que se analiza la implicancia de diferentes políticas monetarias a lo largo del ciclo económico. Todo ello, dentro de un contexto Neo-Keynesiano. Vale decir, que la literatura no cuenta con trabajos que exploren en qué medida la política monetaria afecta la distribución del ingreso, y cómo se comporta este en un contexto de equilibrio general dinámico estocástico dentro de un modelo Neo-Keynesiano.

\subsection{El Modelo Neo-Keynesiano}

El estudio de la macroeconomía puede dividirse en dos ramas: el Neo-Clásico y el NeoKeynesiano ( $N K$ en adelante). El primero se enfoca en la escuela del Ciclo Económico Real y afirma que la causa del ciclo es el carácter estocástico del crecimiento económico. Por su parte, el modelo Neo-Keynesiano incorpora elementos Keynesianos (competencia imperfecta, rigideces nominales, etc.) dentro de un contexto de equilibrio general dinámico similar al de $R B C$. Pueden ser utilizados para analizar la conección entre el dinero, la inflación y el ciclo económico, y para medir la deseabilidad de alternativas monetarias.

Las principales características de esta corriente están dadas por::

1. El modelo $N K$ trae una nueva perspectiva en la naturaleza de la inflación. En primer lugar enfatizan en la naturaleza forward looking de la inflación. Esto aparece claramente reflejado en la Nueva Curva de Phillips (NKPC en adelante).

$$
N K P C: \quad \pi_{t}=(1-\beta) \pi^{*}+\beta E_{t}\left[\pi_{t+1}\right]+\kappa x_{t}
$$

donde $\pi_{t}$ es la inflación, $x_{t}$ la brecha de producción, y los parámetros $0<\beta<1, \kappa>0$ con $\pi^{*}$ la inflación objetivo. Como se puede ver, las expectativas interesan, dado que la inflación es "causada" por una brecha productiva positiva. La autoridad monetaria puede controlar la inflación mediante la brecha productiva. En segundo lugar, el modelo NK hace hincapié en el rol que juegan las variaciones en los markups (o, equivalentemente, en los costos marginales reales) como una fuente de cambio en la inflación agregada.

2. El concepto de brecha productiva juega un papel importante en el modelo de precios rígidos, tanto como fuerza que mueve la inflación (a través de los costos marginales), así como un objetivo de la política.

3. Otro desarrollo del modelo $N K$ es que la transmisión de los shocks de política monetaria no necesariamente implican un efecto de liquides como es tradicionalmente considerado 
en el modelo $I S-L M$. La transmisión ahora se efectúa mediante la tasa de interés.

$$
\text { Curva } I S: \quad \quad x_{t}=E_{t}\left[x_{t+1}\right]-1 / \sigma\left[i_{t}-E_{t}\left[\pi_{t+1}\right]-\bar{r}\right]+\epsilon_{d t}
$$

con $\sigma>0, i_{t}$ la tasa de interés nominal, $\bar{r}$ la tasa libre de riesgo y $\epsilon_{d t}$ el shock de demanda. Una tasa real mayor (ex-ante) de $i_{t}-E_{t}\left[p_{t+1}\right]$ reduce la brecha productiva.

4. La adopción de un contexto de equilibrio general permite un análisis de bienestar y de alternativas políticas monetarias.

5. Una política simple que tienen los Bancos Centrales es la de la tasa de interés en respuesta a variaciones de inflación y/o de la brecha productiva, generalmente provee una aproximación a la regla óptima.

$$
\text { Regla de Taylor: } \quad i_{t}=\left(\bar{r}+\pi^{*}\right)+\phi_{\pi}\left(\pi_{t}-\pi^{*}\right)+\phi_{x} x_{t}+\epsilon_{m t}
$$

$\pi^{*}, \phi_{\pi}, \phi_{x}$ son las elecciones de política, y $\epsilon_{m t}$ el shock de política monetaria. Suponiendo $\pi_{t}>0$, el incremento de inflación aumentan las tasas nominales. La tasa real (ex-post) $i_{t}-p_{t}$ sube con $\phi_{\pi}>1$ (el principio de Taylor). 


\section{Capítulo 3}

\section{El Modelo con agentes heterogéneos}

\subsection{Las Familias}

El modelo original propuesto por Galí y Monacelli [18] será ampliado a uno que contenga agentes heterogéneos dentro de una economía abierta Neo-Keynesiana. A partir de allí, se lo evaluará ante diferentes políticas monetarias, a efectos de analizar los efectos distributivos de la política elegida.

El caso base de nuestro modelo cuenta con diferentes tipos agentes $(h)$ que viven infinitamente, los cuales poseen habilidades distintas entre sí. Todos poseen una determinada dotación de trabajo, quienes buscan maximizar:

$$
E_{0} \sum_{t=0}^{\infty} \beta^{t}\left[U\left(C_{h, t}\right)-V\left(n_{h, t}\right)\right]
$$

donde $C_{h, t}$ corresponde al consumo del individuo $h$ en el momento $t$, y $n_{h, t}$ se refiere a la oferta de trabajo del mismo individuo. Este ultimo factor se divide a su vez en dos componentes: $k_{h}$ denota la productividad individual del agente $h$ y $\tilde{\ell}$ corresponde a un parámetro de ajuste de la oferta de trabajo en la economía. ${ }^{3}$

A su vez, se considera $T_{h}$ como la fracción de familias del tipo $h$, y la población total estará dada por $\sum_{h} T_{h}=1$; por lo que tenemos:

$$
\begin{aligned}
& \sum_{h} T_{h} C_{h, t}=C_{t} \\
& \sum_{h} T_{h} n_{h, t}=\sum_{h} T_{h} k_{h, t} \tilde{\ell}=N_{t}
\end{aligned}
$$

lo cual puede extenderse a un modelo que incluya al agente representativo como se indica en la siguiente expresión :

$$
E_{0} \sum_{t=0}^{\infty} \beta^{t}\left[U\left(C_{t}\right)-V\left(N_{t}\right)\right]
$$

\footnotetext{
${ }^{3}$ Entonces $\tilde{\ell} k_{h}$ denota las unidades de trabajo provisto por el agente $h$.
} 
donde $U$ está dado por $U\left(C_{t}\right) \equiv \frac{C_{t}^{1-\sigma}}{1-\sigma}$, y $V$ as $V\left(N_{t}\right) \equiv \frac{N_{t}^{1+\varphi}}{1+\varphi}$ con $\sigma$ como una constante que indica el grado de aversión al riesgo y $1 / \varphi$ expresa la elasticidad de oferta de trabajo. $C_{t}$ a su vez es un índice de consumo compuesto, definido por:

$$
C_{t}=\left[(1-\alpha)^{\frac{1}{\eta}} C_{H, t}^{\frac{\eta-1}{\eta}}+\alpha^{\frac{1}{\eta}} C_{F, t}^{\frac{\eta-1}{\eta}}\right]^{\frac{\eta}{\eta-1}}
$$

donde $C_{H, t}$ y $C_{F, t}$ representan los índices de consumo de los bienes domésticos e importados respectivamente en el momento $t$. El parámetro $\eta$ denota la elasticidad de substitución entre los bienes domésticos e importados. La maximización de la ecuación (3.2) está sujeta a la siguiente restricción presupuestaria:

$$
\int_{0}^{1}\left[P_{H, t}(i) C_{H, t}(i)+P_{F, t}(i) C_{F, t}(i)\right] d i+E_{t}\left\{Q_{t, t+1} D_{t+1}\right\} \leq D_{t}+W_{t} N_{t}+T_{t}
$$

con $Q_{h, t, t+1}$ (el factor de descuento de un pago nominal), para el cual $E_{t}\left(Q_{h, t, t+1}\right)=1 / R_{t}$, $D_{h, t+1}$ es el pago nominal al individuo en el período $t+1$ de un portfolio al final del período $t$, $W_{t}$ es el salario nominal y $T_{t}$ es un impuesto o transferencia lump-sum, todos estos factores se integran con todas las firmas $i \epsilon[0 ; 1]$

Por lo tanto, la función de utilidad del individuo $h$, expresada de forma detallada, va a estar dado por:

$$
\begin{aligned}
& \underset{\left\{c_{h, t} ; n_{h, t}\right\}_{h=0}^{\infty}}{\operatorname{máx}} E_{0} \sum_{t=0}^{\infty} \beta^{t}\left[\frac{c_{h, t}^{1-\sigma}}{1-\sigma}-\frac{n_{h, t}^{1-\varphi}}{1-\varphi}\right] \\
& \text { s.t. } \int_{0}^{1}\left[P_{H, t}(i) C_{H, h, t}(i)+P_{F, t}(i) C_{F, h, t}(i)\right] d i+E_{t}\left\{Q_{h, t, t+1} D_{h, t+1}\right\} \leq D_{h, t}+W_{t} \underbrace{\tilde{\ell} k_{h, t}}_{n_{h, t}}+T_{h, t}
\end{aligned}
$$

Por su parte, tenemos que $E_{t}\left\{Q_{h, t, t+1} D_{h, t+1}\right\}=D_{h, t}$ y que $\sum_{h} D_{h, t}=D_{t}$.

\subsection{Inflación, Tasa de Cambio Real y los términos de in- tercambio}

En primer lugar, vamos a establecer el índice de precios al consumidor (IPC en adelante), el cual comprende todos los bienes que se tranzan en la economía, tanto domésticos como extranjeros:

$$
P_{t} \equiv\left[(1-\alpha) P_{H, t}^{1-\eta}+\alpha P_{F, t}^{1-\eta}\right]^{\frac{1}{1-\eta}}
$$

con $P_{H, t}$ y $P_{F, t}$ como el índice de precios de los bienes domésticos y extranjeros respectivamente, que a su vez vienen dado por:

$$
P_{j, t} \equiv\left(\int_{0}^{1} P_{j, t}(i)^{1-\varepsilon} d i\right)^{\frac{1}{1-\varepsilon}} \forall j \epsilon\{H, F\} .
$$


En este caso, el parámetro $\varepsilon$ mide la elasticidad de sustitución entre las firmas $i$ dentro del país. El mismo criterio resulta empleable al resto del mundo, con la diferencia de que el resto del mundo importa una muy pequeña cantidad del país analizado, por lo que el coeficiente $\alpha^{*}$ resulta ínfimo, tal que $P_{F, t}^{*}=P_{t}^{*}$.

En este trabajo, se distinguirá la "inflación doméstica" como la tasa de cambio en el índice de precios de los bienes domésticos $\pi_{H, t} \equiv \log \left(\frac{P_{H, t}}{P_{H, t-1}}\right)=\log \left(P_{H, t}\right)-\log \left(P_{H, t-1}\right)$, y la "inflación$I P C^{\prime \prime}$, como la tasa de cambio del $I P C$, por lo que $\pi_{t} \equiv\left(\frac{P_{t}}{P_{t-1}}\right)=\log \left(P_{t}\right)-\log \left(P_{t-1}\right)$. Tal es así que resulta $\pi_{F, t}^{*}=\pi_{t}^{*}$.

Por otra parte, $s_{t}$ denota los "Términos de Intercambio", o sea, el precio de los bienes extranjeros en término a los bienes domésticos, que viene dado por:

$$
s_{t} \equiv\left(\frac{P_{F, t}}{P_{H, t}}\right)=p_{F, t}-p_{H, t},
$$

La "Tasa Nominal" $e_{t}$ se definirá como el precio de la moneda extranjera en términos de la moneda local ,

$$
e_{t}=\log \left(\mathcal{E}_{t}\right) \equiv\left(\frac{P_{F, t}}{P_{F, t}^{*}}\right)=p_{F, t}-p_{F, t}^{*},
$$

En este trabajo, se supone que rige la ley de 1 precio, lo cual implica que $P_{F, t}(i)=e_{t} P_{F, t}^{*}(i)$, para todo $i \epsilon[0,1]$. Ahora, se deriva la relación entre los términos de Intercambio y el Tipo de Cambio Real. Dado nuestra suposición de que la proporción de bienes importados por el resto del mundo es ínfima, y las relaciones $P_{F, t}^{*}=P_{t}^{*}$ y $\pi_{F, t}^{*}=\pi_{t}^{*}$, resulta posible expresar el Tipo de Cambio Real como

$$
q_{t}=\log \left(\mathcal{Q}_{t}\right) \equiv \frac{e_{t} P_{t}^{*}}{P_{t}}=e_{t}+\log \left(P_{t}^{*}\right)-\log \left(P_{t}\right)=e_{t}+p_{t}^{*}-p_{t}
$$

A partir de la últimas dos ecuaciones, resulta que $q_{t}=s_{t} P_{H, t}$, la que log-linealizado resulta $q_{t}=s_{t}+p_{H, t}$. Entonces, utilizando la expresión $p_{H, t}=-\alpha s_{t}$, se obtiene una relación simple entre le tipo de cambio real y los términos de intercambio:

$$
q_{t}=(1-\alpha) s_{t}, \quad \text { lo que implica } \quad p_{t}=p_{H, t}+\alpha s_{t}
$$

\subsection{Las Firmas}

En este modelo, cada firma $i \epsilon[0 ; 1]$ produce $Y_{t}(i)$, con una función de producción $Y_{t}(i)=$ $A_{t} N_{t}(i)$, en el que $\log \left(A_{t}\right)=a_{t}=\rho_{a} a_{t-1}+\epsilon_{t}$ denota la productividad estocástica. La loglinealización de dicha expresión resulta:

$$
y_{t}=n_{t}+a_{t}
$$

Dado el hecho de que este modelo considera los subsidios de desempleo $\tau$, el beneficio de las firmas por unidad de producción viene dado por $P_{t}(i) Y_{t}(i)-(1-\tau) W_{t} N_{t}(i) / A_{t}$. De este modo, 
los costos marginales se representan mediante $M C_{t}^{n}=(1-\tau) W_{t} / A_{t}$. El modelo presentado por Galí y Monacelli [18] contiene firmas que poseen poder de mercado, por lo que los precios se establecen superiores a los costos marginales, y su mark-up. Tal es así, que bajo la teoría de Calvo, la proporción $1-\theta$ de un grupo de firmas aleatoriamente seleccionadas fijan los nuevos precios cada período. Como se demuestra en el Apéndice A.1, la estrategia óptima de fijación de precios para la firma en el período $t$ se puede aproximar en un contexto de inflación cero para el steady state mediante la regla:

$$
\bar{P}_{H, t}=\mu+(1-\beta \theta) \sum_{k=0}^{\infty}(\beta \theta)^{k} E_{t}\left\{m c_{t+k}^{n}\right\}
$$

donde $\bar{P}_{H, t} \equiv \log \left(\frac{P_{H, t}^{n e w}}{P_{H, t}}\right)$ es el porcentaje de desviación del nuevo precio de bienes domésticos, representado por $P_{H, t}^{n e w}$ a partir de índice de precios domésticos $P_{H, t}$ en el período $t, \mathrm{y}-\mu=$ $-\log \left(\frac{\varepsilon}{\varepsilon-1}\right)$ representa el markup que sería obtenido en una situación de precios flexibles. Las firmas en el resto del mundo enfrentan una situación similar, donde la productividad surge a partir de $\log \left(A_{t}^{*}\right)=a_{t}^{*}=\rho_{a}^{*} a_{t-1}^{*}+\epsilon_{t}^{*}$.

Más aún, la productividad individual sigue el siguiente proceso estocástico.

$$
\log k_{h, t}=\log a_{t}+\log g_{h}+u_{h, t}
$$

siendo $u_{h, t}$ el shock de productividad individual específico y $g_{h}$ denota la productividad en steady state del individuo $h$ en el momento $t$.

\subsection{Algunas Identidades}

La canasta óptima de cada individuo está dada por:

$$
c_{h, j, t}(i)=\left(\frac{P_{j, t}(i)}{P h, j, t}\right)^{-\varepsilon} c_{h, j, t} \quad \forall j \epsilon\{H, F\}
$$

para todo $i \epsilon[0,1]$, donde $P_{H, t} \equiv\left(\int_{0}^{1} P_{H, t}(i)^{1-\varepsilon} d i\right)^{\frac{1}{1-\varepsilon}}$ y $P_{F, t} \equiv\left(\int_{0}^{1} P_{F, t}(i)^{1-\varepsilon} d i\right)^{\frac{1}{1-\varepsilon}}$ son todos los indices de precios de bienes domésticos e importados, ambos expresados en moneda local. La distribución óptima entre los bienes locales y extranjeros implica:

$$
C_{h, H, t}=(1-\alpha)\left(\frac{P_{H, t}}{P_{t}}\right)^{1-\eta} C_{h, t} \quad ; \quad C_{h, F, t}=\alpha\left(\frac{P_{F, t}}{P_{t}}\right)^{1-\eta} C_{h, t}
$$

Como se demuestra en el Apéndice A.2, de la ecuación (A.8) podemos obtener la ecuación estándar intra-temporal que relaciona la utilidad marginal del trabajo y consumo con el salario real:

$$
c_{h, t}^{\sigma} N_{t}^{\varphi}=\frac{W_{t}}{P_{t}}
$$


De la ecuación (A.9) obtenemos la relación de Euler:

$$
\beta R_{t} E_{t}\left\{\left(\frac{c_{h, t+1}}{c_{h, t}}\right)^{-\sigma}\left(\frac{P_{t}}{P_{t+1}}\right)\right\}=1
$$

En el Apéndice A.2.2 se derivan dos ecuaciones que serán utilizadas a lo largo de este trabajo:

$$
\begin{aligned}
& \sigma c_{h, t}+\varphi \widehat{\theta}_{h, t}=w_{t}-p_{t} \\
& c_{h, t}=E_{t}\left[c_{h, t+1}\right]-\frac{1}{\sigma}\left[-\rho+r-E_{t}\left\{\pi_{t+1}\right\}\right]
\end{aligned}
$$

donde se define $\rho \equiv-\log \beta$.

\subsection{Riesgo Internacional Compartido}

En el resto del mundo, los agentes enfrentan un problema similar al establecido para la economía del país pequeño. Con respecto a las preferencias, sin embargo, la ponderación de los bienes producidos en la economía pequeña, representadas por $\alpha^{*}$ resulta irrelevante. Bajo el supuesto de mercados completos de "securities", la condición de primer orden análoga a la ecuación (3.18) para el agente del resto del mundo:

$$
\beta E_{t}\left\{\left(\frac{C_{t+1}^{*}}{C_{t}^{*}}\right)^{-\sigma}\left(\frac{P_{t}^{*}}{P_{t+1}^{*}}\right)\left(\frac{e_{t}}{e_{t+1}}\right)\right\}=Q_{t, t+1}
$$

Combinando las ecuaciones (3.18) y (3.21) y haciendo uso del tipo de cambio real $\left(q_{t} \equiv \frac{e_{t} P_{t}^{*}}{P_{t}}\right)$ se obtiene:

$$
\left(\frac{C_{t+1}^{*}}{C_{t}^{*}}\right)^{-\sigma}\left(\frac{P_{t}^{*} e_{t}}{P_{t}}\right)\left(\frac{P_{t+1}}{P_{t+1}^{*} e_{t+1}}\right)=\left(\frac{C_{t+1}}{C_{t}}\right)^{-\sigma}
$$

que después de iterar resulta:

$$
C_{t}=\vartheta C_{t}^{*} \mathcal{Q}_{t}^{\frac{1}{\sigma}}
$$

donde $\vartheta=\frac{\alpha^{*}}{\alpha}$ es la relación de las importaciones de las dos economías. Como se demuestra en la ecuación(A.13), se obtiene:

$$
c_{t}=c_{t}^{*}+\left(\frac{1-\alpha}{\sigma}\right) s_{t}
$$

Tal como lo expresan Galí y Monacelli [18], el supuesto de mercados completos a nivel internacional resulta en una simple relación entre el consumo local y extranjero y el tipo de cambio real, todo en desvío porcentual del steady-state. Esa relación es independiente del tamaño relativo de la dos economías. 


\subsection{El Equilibrio}

\subsubsection{Consumo mundial y su producción}

Para empezar, se describe como el consumo y la producción se determinan en la economía mundial. Dado el hecho que las preferencias de las familias son similares, pero son una ínfima ponderación en los bienes importados desde la economía pequeña, se puede expresar la condición de mercado como $y_{t}^{*}=c_{t}^{*}$, y recordando la ecuación de Euler del consumidor en la economía pequeña (ecuación (3.20)), que también rige para la economía mundial:

$$
c_{t}^{*}=E_{t}\left[c_{t+1}^{*}\right]-\frac{1}{\sigma}\left[-\rho+r^{*}-E_{t}\left\{\pi_{t+1}^{*}\right\}\right]
$$

a partir de la relación anterior

$$
y_{t}^{*}=E_{t}\left[y_{t+1}^{*}\right]-\frac{1}{\sigma}\left[-\rho+r^{*}-E_{t}\left\{\pi_{t+1}^{*}\right\}\right]
$$

\subsubsection{Consumo y Producción en la Economía Pequeña}

La condición de mercado en la economía pequeña requiere que $Y_{t}(i)=C_{H, t}(i)+C_{H, t}^{*}(i)$, para todo $i \epsilon[0,1]$. La log-linealización en el steady-state implica:

$$
y_{t}(i)=(1-\alpha) c_{H, t}(i)+\alpha c_{H, t}^{*}(i)
$$

Dado $Y_{t} \equiv \int_{0}^{1} Y_{t}(i) d i=Z_{t} \int_{0}^{1} N_{t}(i) Z_{t} N_{t}$ que denota la producción doméstica agregada. Loglinealizando en el steady-state se obtiene $y_{t}=\int_{0}^{1} y_{t}(i) d i=z_{t}+n_{t}$. Del mismo modo, la loglinealización de $C_{H, t}$ y $C_{H, t}^{*}$ resulta $c_{H, t}=\int_{0}^{1} c_{H, t}(i) d i$ y $c_{H, t}^{*}=\int_{0}^{1} c_{H, t}^{*}(i) d i$, respectivamente. A partir de estas expresiones se obtiene:

$$
y_{t}=(1-\alpha) c_{H, t}+\alpha c_{H, t}^{*}
$$

Combinando esta ultima expresión, con la log-linealización de las ecuaciones (3.3) y (3.15) obtenemos:

$$
\begin{aligned}
y_{t} & =c_{t}+\alpha\left(c_{H, t}^{*}-c_{F, t}\right) \\
& =(1-\alpha) c_{t}+\alpha c_{t}^{*}+\alpha\left[\left(c_{H, t}^{*}-c_{t}^{*}\right)-\left(c_{F, t}-c_{t}\right)\right]
\end{aligned}
$$

A partir de las relaciones expresadas en 3.4 y 3.2 se tiene que $c_{F, t}-c_{t}=-\eta p_{F, t}=-\eta(1-\alpha) s_{t}$ (dado el supuesto de $P_{t}^{*}=P_{F, t}^{*}$ ), por lo que se puede reexpresar la última ecuación como:

$$
y_{t}=(1-\alpha) c_{t}+\alpha c_{t}^{*}+\alpha \eta(2-\alpha) s_{t}
$$

Lo que la ecuación (3.25) indica es que la producción es determinada como un promedio ponderado del gasto doméstico y extranjero, más un "factor de gasto" que resulta proporcional a los términos de intercambio, todo ello en desvío porcentual de su steady-state (ver Galí y Monacelli 
$[18])$.

Además, se pueden combinar (3.23), (3.25), y la relación $y_{t}^{*}=c_{t}^{*}$ para obtener:

$$
y_{t}=(1-\alpha)\left[c_{t}^{*}+\frac{1}{\sigma} q_{t}\right]+\alpha c_{t}^{*}+\alpha \eta(2-\alpha) s_{t}
$$

y tras algunas manipulaciones algebraicas:

$$
y_{t}=y_{t}^{*}+\frac{\omega_{\alpha}}{\sigma} s_{t}
$$

donde $\omega_{\alpha} \equiv 1+\alpha(2-\alpha)(\sigma \eta-1)$. Ahora resulta posible reemplazar los términos de intercambio por el consumo en la última expresión:

$$
y_{t}=y_{t}^{*}+\frac{\omega_{\alpha}}{\sigma}\left(c_{t}-c_{t}^{*}\right) \frac{\sigma}{1-\alpha}
$$

que tras ordenar resulta:

$$
c_{t}=\Phi_{\alpha} y_{t}+\left(1-\Phi_{\alpha}\right) y_{t}^{*} \quad \text { con } \quad \Phi_{\alpha} \equiv \frac{1-\alpha}{\omega_{\alpha}}>0
$$

Finalmente, las exportaciones netas se expresan como $n x_{t} \equiv\left(\frac{1}{Y}\left(Y_{t}-\frac{P_{t}}{P_{H, t}} C_{t}\right)\right.$ el cual puede ser expresado tras aproximación mediante $n x_{t}=y_{t}-c_{t}-\alpha s_{t}$, luego combinándolo con 3.26 y 3.27 se obtiene:

$$
\begin{aligned}
n x_{t} & =\left(y_{t}-y_{t}^{*}\right)\left[(1-\alpha)-\frac{\alpha \sigma}{\omega_{\alpha}}\right] \\
& =\frac{\alpha \Lambda}{\omega_{\alpha}}\left(y_{t}-y_{t}^{*}\right),
\end{aligned}
$$

donde $\Lambda \equiv(2-\alpha)(\sigma \eta-1)(1-\sigma)$

\subsection{La Oferta: Dinámica Inflacionaria, Costos Marginales y la Brecha de Producción}

Como se demuestra en el Apéndice A.1, la dinámica inflacionaria tanto en la economía pequeña y el resto del mundo están dados por:

$$
\begin{aligned}
\pi_{H, t} & =\beta E_{t}\left\{\pi_{H, t+1}\right\}+\lambda\left(m c_{t}+\mu\right) \\
\pi_{t}^{*} & =\beta E_{t}\left\{\pi_{t+1}^{*}\right\}+\lambda\left(m c_{t}^{*}+\mu\right)
\end{aligned}
$$

donde $\lambda \equiv \frac{(1-\theta)(1-\beta \theta)}{\theta}$. Para poder analizar los costos marginales, proseguimos de la siguiente manera: en primer lugar, recordemos de la sección 3.3 que $M C_{t}^{n}=M C_{t} P_{H, t}$ por lo tanto, tomando logaritmos para la economía pequeña y el resto del mundo tenemos:

$$
\begin{aligned}
& m c_{t}=-\nu+w_{t}-a_{t}-p_{H, t} \\
& m c_{t}^{*}=-\nu^{*}+w_{t}^{*}-a_{t}^{*}-p_{t}^{*}
\end{aligned}
$$


En el Apéndice A.3 se derivan los costos marginales en relación con $y_{t}, y_{t}^{*}, a_{t}$ y $a_{t}^{*}$, obteniendo las siguientes expresiones que indican los costos marginales para ambas economías:

$$
\begin{aligned}
& m c_{t}=-\nu+\left(\frac{\sigma}{\omega_{\alpha}}+\varphi\right) y_{t}+\sigma\left(1-\frac{1}{\omega_{\alpha}}\right) y_{t}^{*}-(1+\varphi) a_{t} \\
& m c_{t}^{*}=-\nu^{*}+(\sigma+\varphi) y_{t}^{*}-(1+\varphi) a_{t}^{*}
\end{aligned}
$$

A continuación definimos la brecha de producción como el desvío de la producción efectiva de su nivel natural, lo que puede ocurrir bajo precios flexibles y por lo tanto costos marginales constantes $m c_{t}=m c^{*}=-\mu$, por lo que tenemos $\tilde{y}_{t} \equiv y_{t}-\bar{y}_{t}$ y $\tilde{y}_{t}^{*} \equiv y_{t}^{*}-\bar{y}_{t}^{*}$, resolviendo para la producción doméstica en las ecuaciones (3.33) y (3.34):

$$
\begin{aligned}
& \bar{y}_{t}=\Omega_{\alpha}+\Gamma_{\alpha} a_{t}+\Theta_{\alpha} y_{t}^{*} \\
& \bar{y}_{t}^{*}=\Omega_{0}+\Gamma_{0} a_{t}^{*}
\end{aligned}
$$

en las expresiones anteriores, se define $\Omega_{\alpha}=\frac{\omega_{\alpha}(\nu-\mu)}{\sigma+\varphi \omega_{\alpha}}, \Gamma_{\alpha}=\frac{\omega_{\alpha}(1+\varphi)}{\sigma+\varphi \omega_{\alpha}}, \Theta_{\alpha}=\frac{\sigma\left(1-\omega_{\alpha}\right)}{\sigma+\varphi \omega_{\alpha}}, \Omega_{0}=\frac{\nu^{*}-\mu}{\sigma+\varphi}$ y $\Gamma_{0}=\frac{1+\varphi}{\sigma+\varphi} a_{t}^{*}$.

\subsection{Dinámica de Equilibrio}

Las ecuaciones A.19 y A.20 establecen los costos marginales de ambas economías, las cuales combinadas con 3.29 y 3.30 resultan en:

$$
\begin{aligned}
& \pi_{H, t}=\beta E_{t}\left\{\pi_{H, t+1}\right\}+\kappa_{\alpha} \tilde{y}_{t} \\
& \pi_{t}^{*}=\beta E_{t}\left\{\pi_{t+1}^{*}\right\}+\kappa_{0} \tilde{y}_{t}^{*}
\end{aligned}
$$

donde $\kappa_{\alpha} \equiv \lambda\left(\frac{\sigma}{\omega_{\alpha}}+\varphi\right)$ y $\kappa_{0} \equiv \lambda(\sigma+\varphi)$. Estas dos ecuaciones representan la $N K P C$ tanto de la economía local y mundial. En el Apéndice A.3 se derivan las ecuaciones $I S$ de ambas economías:

$$
\begin{aligned}
& \tilde{y}_{t}=E_{t}\left\{\tilde{y}_{t+1}\right\}-\frac{\omega_{\alpha}}{\sigma}\left(r_{t}-E_{t}\left\{\pi_{H, t+1}\right\}-\overline{r r}_{t}\right) \\
& \tilde{y}_{t}^{*}=E_{t}\left\{\tilde{y}_{t+1}^{*}\right\}-\frac{1}{\sigma}\left(r_{t}^{*}-E_{t}\left\{\pi_{t+1}^{*}\right\}-\overline{r r}_{t}^{*}\right)
\end{aligned}
$$

donde $\overline{r r}_{t} \equiv \rho-\frac{\sigma(1+\varphi)\left(1-\rho_{\alpha}\right)}{\sigma+\omega_{\alpha \varphi}} a_{t}-\varphi \Theta_{\alpha} E_{t}\left\{\Delta y_{t+1}^{*}\right\} ;$ y $\overline{r r}_{t}^{*} \equiv-\sigma \Gamma_{0} a_{t}^{*}\left(1-\rho_{\alpha}^{*}\right)+\rho$ que representan la tasa natural de interés de ambas economías.

\subsection{Políticas Monetarias Alternativas}

\subsubsection{Economía Mundial}

En este trabajo se considera a la política monetaria mundial esóptima, de este modo, existe una producción y una tasa de inflación mundial estables. Por lo que se establece: $\tilde{y}_{t}=\pi_{t}^{*}=0$. Estas últimas dos expresiones conducen la tasa de interés a su nivel natural, por lo que se obtiene:

$$
r_{t}^{*}=\rho-\sigma\left(1-\rho_{a}^{*}\right) \Gamma_{0} a_{t}^{*}
$$




\subsubsection{La Economía Abierta}

La economía abierta plantea tres políticas monetarias alternativas, a efectos de analizar los impactos distributivos en la economía doméstica. La economía abierta puede estabilizar la inflación de los bienes domésticos (objetivo de inflación doméstica -OID- en adelante), podría estabilizar la inflación $I P C$, o puede formar parte de una unión monetaria con el resto del mundo, fijando el tipo de cambio a la moneda mundial $(O I F)$.

\section{Objetivo de Inflación Doméstica}

La primera política monetaria a considerar es la que apunta a controlar la inflación doméstica $(O I D)$. Por lo tanto, se la considera igual cero para todos los períodos, por lo que tenemos $\widetilde{y}_{t}=\pi_{H, t}=0$; por lo que no hay brecha de producción en la economía. De la ecuación (3.39), tenemos que la tasa de interés alcanza su nivel natural:

$$
r_{t}=\overline{r r}_{t}
$$

De la ecuaciones (3.9) y (3.10) tenemos que $s_{t}=e_{t} \Rightarrow P_{H, t}=P_{F, t}^{*}$, por lo que:

$$
s_{t}=q_{t}\left(\frac{1}{1-\alpha}\right)=\frac{\sigma(1+\varphi)}{\sigma+\omega_{\alpha} \varphi}\left(a_{t}-a_{t}^{*}\right)
$$

del mismo modo, de la ecuación(3.11), el nivel de precios $I P C$ viene dado por:

$$
p_{t}=\alpha e_{t}=\frac{\alpha \sigma(1+\varphi)}{\sigma+\omega_{\alpha \varphi}}\left(a_{t}-a_{t}^{*}\right)
$$

dado la constancia de los precios domésticos y extranjeros. Bajo esta política, el nivel IPC varia con el diferencial de productividad y adopta sus propiedades estadísticas. Lo mismo sucede con el tipo de cambio real, que viene dado por $q_{t}=(1-\alpha) e_{t}$.

Bajo esta política la producción, la economía siempre crecerá en respuesta a un shock tecnológico (a partir de la ecuación (3.35)). La respuesta a un incremento de la producción mundial depende de $\omega_{\alpha}$. Es negativa si $\omega_{\alpha}>1$, y positiva en el otro caso.

\section{Objetivo de Inflación IPC}

Si el objetivo de la política monetaria es el de estabilizar el índice de precios al consumidor tenemos $\pi_{t}=0$; por lo que $p_{t}=p_{t}^{*}=0$ de la ecuación (3.11) tenemos:

$$
p_{H, t}=-\alpha s_{t}
$$

Lo cual implica una dinámica común para el nivel de precios domésticos y los términos de intercambio. En el Apéndice se deriva la ecuación de diferencias de segundo orden estocástica $p_{H, t}$ :

$$
\gamma_{c} p_{H, t}=p_{H, t-1}+\beta E_{t}\left\{p_{H, t+1}\right\}-\lambda(1+\varphi)\left(a_{t}-a_{t}^{*}\right)
$$


donde $\gamma_{c} \equiv 1+\beta+\frac{\lambda}{\alpha}\left(1+\frac{\varphi \omega_{\alpha}}{\sigma}\right)$, la cual puede ser resuelta suponiendo igualdad entre la autocorrelación de los shocks de productividad tanto locales como mundial, resultando la siguiente ecuación:

$$
p_{H, t}=\xi_{c} p_{H, t-1}-\zeta_{c}\left(a_{t}-a_{t}^{*}\right)
$$

$\operatorname{con} \xi_{c} \equiv \frac{1}{2 \beta}\left(\gamma_{c}-\sqrt{\gamma_{c}^{2}-4 / \beta}\right) \epsilon(0,1)$ y $\zeta_{c}=\frac{\lambda \xi_{c}(1+\varphi)}{\left(1-\xi_{c} \beta \rho_{a}\right)}>0$

Resulta entonces que un cambio en la productividad relativa conlleva a una respuesta negativa de los precios domésticos. La razón es que si se incrementa la productividad doméstica, existe una depreciación de los términos de intercambio, lo que lleva a una inflación OI-IPC. La estabilización de esto, se logra entonces mediante una reducción de los precios domésticos y una pequeña depreciación, lo que puede obtenerse con una brecha de producción negativa.

Dado (3.45)

$$
e_{t}=q_{t}=-\frac{1-\alpha}{\alpha} p_{H, t}
$$

el tipo de cambio nominal resulta estacionario, y dado la evolución del índice de precios doméstico, se deprecia en respuesta a un aumento de productividad. A diferencia del modelo OID, tanto el tipo de cambio nominal como real presentan una persistencia.

\section{Fijación $P E G$}

El modelo de tipo de cambio adoptado por la autoridad monetaria corresponde a la decisión del país de formar parte de una unión monetaria, donde la economía local sigue la política monetaria de la economía mundial. El único instrumento disponible por la economía local es el de la tasa de interés, por lo que se obtiene un tipo de cambio nominal constante (por simplicidad, se lo establecerá igual a cero). Por lo tanto, tenemos que $s_{t}=-p_{H, t}$ y que $q_{t}=-p_{t}$, para el período $t$.

Empleando la misma estrategia que en el caso anterior, resulta posible obtener la ecuación de diferencia de segundo orden para el nivel de precios doméstico, o sea:

$$
\gamma_{e} p_{H, t}=p_{H, t-1}+\beta E_{t}\left\{p_{H, t+1}\right\}-\lambda(1+\varphi)\left(a_{t}-a_{t}^{*}\right)
$$

donde $\gamma_{e} \equiv 1+\beta+\lambda\left(1+\frac{\varphi \omega_{\alpha}}{\sigma}\right)$. Suponiendo $\rho_{a}=\rho_{a}^{*}$, esta ecuación presenta la siguiente representación estacionaria

$$
p_{H, t}=\xi_{e} p_{H, t-1}-\zeta_{e}\left(a_{t}-a_{t}^{*}\right)
$$

$\operatorname{con} \xi_{e} \equiv \frac{1}{2 \beta}\left(\gamma_{e}-\sqrt{\gamma_{e}^{2}-4 / \beta}\right) \epsilon(0,1)$ y $\zeta_{e}=\frac{\lambda \xi_{e}(1+\varphi)}{\left(1-\xi_{e} \beta \rho_{a}\right)}>0$

En este caso, la respuesta de los precios domésticas es similar al del caso OI-IPC. Más aún, tiende a demostrar una persistencia ante la variación de productividad diferencial. Esto resulta porque: siendo $\gamma_{e}<\gamma_{c}$, entonces $\xi_{e}>\xi_{c}$ y $\zeta_{e}>\zeta_{c}$. Tal es así, que la política OIF conlleva a un ajuste mas fuerte de los precios domésticos (y por lo tanto de la brecha productiva) en 
respuesta a un shock de productividad diferencial.

Siendo $p_{t}^{*}=e_{t}=0$ resulta

$$
p_{t}=(1-\alpha) p_{H, t}
$$

A diferencia del modelo $O I D$, el $O I-I P C$ debe bajar en respuesta a un incremento de productividad.

\subsection{Dinámica Distributiva}

En este trabajo, utilizamos el coeficiente de Gini a efectos de analizar la dinámica en la distribución del ingreso. El mismo se define como el área entre la línea de perfecta igualdad y la curva de Lorenz con respeto a la línea de perfecta igualdad. La figura 3.1 muestra la línea de perfecta igualdad, así como la curva de Lorenz. De acuerdo a la definición presentada, el coeficiente de Gini está dado por $A /(A+B)$ o lo que es lo mismo $1-2 B$ dado que $A+B=0,5$. Resulta importante notar que el coeficiente de Gini se encuentra en el rango $[0,1]$. Un valor próximo a cero indica perfecta igualdad, en tanto que un valor cercano a uno corresponde a una situación donde una persona recibe todo el ingreso.

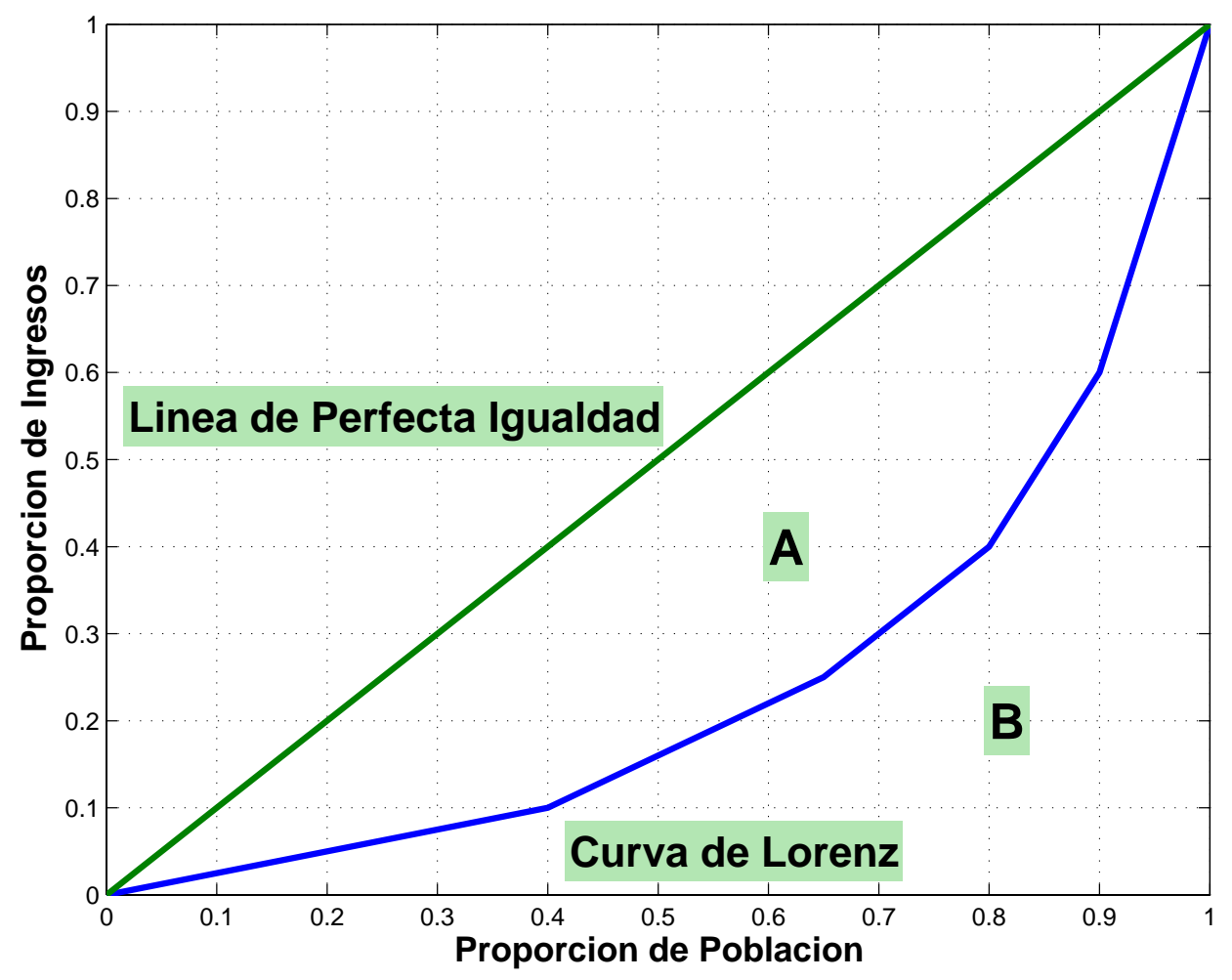

Figura 3.1: Curva de Lorenz.

La dinámica del coeficiente de Gini en nuestro modelo se calculará de la siguiente manera. 
En primer lugar, definimos $£$ como la fracción de ingreso del agente de tipo $h$ y la suma acumulada de $£, \triangle$, tal que:

$$
\begin{aligned}
£_{h, t} & =\frac{W_{t}^{r} n_{h, t}+r_{t} D_{h, t}}{¥+D_{t+1}} \\
¥ & =\sum_{h=1}^{i} W_{t}^{r} n_{h, t} ; \quad D_{t}=\sum_{h=1}^{i} D_{h, t} \\
\triangle_{h, t} & =\sum_{h=1}^{i} £_{h, t}
\end{aligned}
$$

De este modo, la línea debajo de la curva de Lorenz y el correspondiente coeficiente de Gini se pueden calcular de la siguiente manera:

$$
\begin{aligned}
& B_{t}=\sum_{h} T_{h} \triangle_{h, t}-0,5 \sum_{h} T_{h} £_{h, t} \\
& \text { Gini }_{t}=1-2 B_{t}
\end{aligned}
$$

donde $B_{t}$ es el área debajo de la curva de Lorenz en el momento $t$. Posteriormente, se definen las ecuaciones de steady state de las expresiones anteriores y se las log-linealiza, para obtener:

$$
\begin{aligned}
& \bar{£} \widehat{£}_{h, t}=\frac{\overline{W k_{h}}}{\bar{P}}\left(\widehat{W}_{t}+\widehat{k}_{h, t}-\widehat{p}_{T}\right)+\bar{r} \overline{D_{h}}\left(\widehat{r}_{t}+\widehat{D}_{h, t}\right)-\frac{\overline{W N_{h}}}{\bar{P}} \widehat{\Psi}-\bar{D} \widehat{D}_{t+1} \\
& \bar{\triangle} \widehat{\triangle}_{h, t}=\sum_{h=1} \bar{£}_{h} \widehat{£}_{h, t} \\
& \bar{B} \widehat{B}=\sum_{h} T_{h} \bar{\triangle}_{h} \widehat{\triangle}_{h, t}-0,5 \sum_{h} T_{h} \bar{£}_{h} \widehat{£}_{h, t} \\
& \overline{\text { Gini Gini }}=-2 \bar{B} \widehat{B}_{t}
\end{aligned}
$$

Cabe aclarar, que las variables que contienen una barra superior corresponden a su valor de steady state, en tanto que el sombrero indica la log-linealización de dicha variable. 


\section{Capítulo 4}

\section{Resultados}

En esta sección, se presentan los resultados y las funciones de reacción del modelo ante los diferentes shocks con sus correspondientes explicaciones. En primer lugar, pasamos a implementar la calibración a efectos de analizar los resultados. El parámetro $\beta$ se establece en 0.987 siguiendo a Cooley y Prescott [12]. La elasticidad de substitución entre los bienes domésticos y extranjeros $(\eta)$ es de 1.5, de acuerdo a Backus [13], notar que el trabajo de Galí y Monacelli [18] lo establecen en la unidad. Para la correlación de productividad, así como para el grado de apertura, utilizamos los datos de Galí y Monacelli [18]. Ellos establecen que $\mu$ es de alrededor de $20 \%$, lo cual coincide con las investigaciones de Rotenberg y Woodford [17], así como con las de Schmitt-Grohé y Uribe [16]. Según Galí y Monacelli [18], tomamos $\mu=0,182$, por lo que aplicando la relación $-\mu=-\log \left(\frac{\varepsilon}{\varepsilon-1}\right)$, resulta $\varepsilon=6$. Del mismo modo, fijamos $\alpha=0,40$, $\alpha^{*}=0,001, \Theta=0,75, \Theta^{*}=0,75,1 / \varphi=1 / 3$ y $\sigma=1$. Siguiendo a Cooley y Prescott [12], el desvío estándar del shock de productividad doméstica y extranjera viene dado por $\sigma_{\epsilon}=0,007$. En tanto que la autocorrelación de la productividad $\rho_{a} \mathrm{y} \rho_{a}^{*}$ es igual a 0.95.

\section{Cuadro 4.1: Calibración}

\begin{tabular}{lrl}
\hline \hline Parámetro & Valor & Explicación \\
\hline$\beta$ & 0.987 & Factor de descuento \\
$\eta$ & 1.5 & Elasticidad de substitución entre los bienes domésticos y extranjero \\
$\mu$ & 0.182 & Logaritmo del markup \\
$\varepsilon$ & 6.00 & Elasticidad de substitución entre bienes de cada categoría \\
$\sigma$ & 1.00 & Grado de aversión al riesgo \\
$\varphi$ & 3.00 & Inversa de la elasticidad de oferta de trabajo \\
$\alpha$ & 0.40 & Grado de apertura de la economía pequeña \\
$\alpha^{*}$ & 0.001 & Grado de apertura de la economía mundial \\
$\Theta$ & 0.75 & Porcentaje de firmas nacionales que no pueden re(set) precios en el período $t$ \\
$\Theta^{*}$ & 0.75 & Porcentaje de firmas extranjeras que no pueden re(set) precios en el período $t$ \\
$\sigma_{\epsilon}$ & 0.007 & Desvío estándar del shock de productividad doméstica \\
$\rho_{a}$ & 0.95 & autocorrelación de la productividad nacional \\
$\rho_{a}^{*}$ & 0.95 & autocorrelación de la productividad internacional \\
\hline \hline
\end{tabular}


En esta sección, se tomará como punto de partida una economía en la que existen 2 tipos de consumidores. El consumidor tipo 1 (o del sector 1 como también se lo puede enunciar) representa el $80 \%$ de la población que percibe el $20 \%$ del ingreso nacional (compuesto de ingresos laborales y percepción de rentas). El consumidor tipo 2 representa el $20 \%$ de la población y percibe el $80 \%$ del ingreso nacional. Por su parte, el consumidor tipo 1 destina solo el $10 \%$ de su ingreso a bienes importados, en tanto que el consumidor tipo 2 destina $30 \%$ de su ingreso.

\subsection{Comportamiento Agregado de la Economía}

\subsubsection{Shock de Productividad Doméstica Agregada}

Un shock de productividad doméstica $(a)$, como era de esperarse, lleva a un incremento mayor de la producción local $(y)$, lo cual a su vez conlleva a un aumento de la exportaciones (a partir de 3.28) y un menor crecimiento del consumo local agregado (c). Este último fenómeno, se debe en parte a que una porción del consumo de las familias está compuesto por bienes extranjeros. A partir del hecho que el shock es de carácter doméstico, y que nuestra economía en cuestión es pequeña, tanto la producción, como el consumo extranjero $\left(y^{*}\right.$ y $\left.c^{*}\right)$ permanecen invariantes.

\section{Objetivo de Inflación Doméstica - (OID)}

Cabe recordar, que cuando la política monetaria fijada por la pequeña economía es de carácter $O I D$, tenemos que $p_{H}$ y $\pi_{H}$ son iguales a cero por definición, por lo que la brecha de producción $\widetilde{y}_{t}=0$. Bajo este contexto, un shock de productividad local provoca un aumento del tipo de cambio nominal $e_{t}$, lo que a su vez hace que los bienes extranjeros se vuelvan más caros. Por tal motivo, resulta posible explicar el porqué de la disminución en el consumo de bienes extranjeros. Por otro lado, se observa que ante un incremento en el diferencial tecnológico, el nivel de precios $I P C$ se incrementa, en tanto que la inflación $\pi_{t}$ experimenta un incremento en primer lugar, en tanto que después empieza a decaer (incluso tomando valores negativos). Por último, el interés nominal presenta una pequeña caída. Todas estas conclusiones se observan en la Figura 4.1a

Este shock doméstico, incrementa la productividad del trabajo, lo que como ya se dijo conlleva a una reacción positiva del producto. Sin embargo, dado que el aumento de productividad es mayor que el del producto, las horas trabajadas deben reducirse dado que las firmas no pueden incrementar la producción. Este resultado va en línea con la literatura Neo Keynesiana. Estos últimos resultados resultan interesantes para analizar las consecuencias distributivas. En primer lugar, dado que el aumento del salario resulta mayor que la caída de las horas trabajadas, el ingreso de las familias proveniente del trabajo experimenta un aumento, y dado que para la mayor parte de la población el salario constituye su principal fuente de ingreso, la desigualdad tiende a caer. Por otra parte, dado el importante aumento que experimentan tanto el $p_{t}$ como el $\pi_{t}$, el salario real de las familias cae de manera considerable, por lo que el incremento anterior se diluye por este motivo. En consecuencia, la desigualdad se incrementa, para luego empezar a caer, e ir alcanzado su valor original de steady state. A partir del hecho que el ingreso de las 
familias se incrementa, el consumo en general experimenta un comportamiento similar pero en menor medida que el producto. Sin embargo, este aumento está dado principalmente por un aumento del consumo de bienes domésticos, toda vez que la inflación de bienes domésticos es cero, sin embargo, siendo que el sector 2 comprende a una pequeña parte de la economía y que da una gran ponderación a los bienes extranjeros, resulta más afectada que el sector 1, dado que los bienes extranjeros experimentaron un aumento.

\section{Objetivo de Inflación $I P C$ - $(O I-I P C)$}

Bajo este objetivo de política monetaria, resulta que $\pi_{t}=p_{t}=p_{t}^{*}=0$ para todo $t$; pot tal motivo, los precios domésticos, presentan una reacción negativa como surge de (3.47), a partir de la depreciación de los términos de intercambio, en tanto que los precios IPC están estabilizados. La estabilización de la inflación $O I-I P C$ se logra reduciendo los precios domésticos, lo cual se puede obtener con una brecha de producción negativa. Tanto el tipo de cambio nominal como real $\left(e_{t} \mathrm{y} q_{t}\right)$ resultan iguales, y presentan un leve aumento. Considerando la $N K P C$, la tasa de interés nominal presenta una reducción en los primeros períodos, pero luego empieza estabilizarse. Las reacciones de $y, c$ y $n x$ resultan similares al caso anterior (ver Figura 4.2a). Sin embargo, dado el menor aumento del tipo de cambio, el consumo de bienes extranjeros no se reduce tanto, en tanto que las personas que se encuentran en el sector 2 de la economía se ven afectados por el incremento de la tasa de interés, lo que todo esto sumado conlleva a una reducción de la desigualdad.

\section{Fijación PEG - OIF}

Las respuestas de las variables ante un shock de productividad resultan casi similares a las establecidas para la política OID. Sin embargo, resultan muy importantes los resultados respecto al nivel de precios tanto domésticos como nacionales. Se puede observar, que $\downarrow p_{h}>\downarrow$ $p_{t}>\downarrow \pi_{h}>\downarrow \pi_{t}$. Como se explica en la siguiente sección, estos resultados presentan conclusiones importantes con respecto a la distribución del ingreso, toda vez, que este modelo es el que lleva a una mayor reducción de la inflación, y por ende (bajo este planteamiento), a una consecuente reducción de la desigualdad (ver Figura 4.3a). Con esta política monetaria, ante un shock de carácter doméstico, los bienes locales se abaratan considerablemente en comparación con los extranjeros, lo que produce consecuentemente una deflación tanto de los precios de bienes domésticos como del nivel de precios IPC. Por tal motivo, el tipo de cambio real debe subir, a efectos de equiparar la situación de la política monetaria de la economía pequeña con la del resto del mundo.

\section{Situación de los Consumidores}

Ante un shock de productividad doméstico, los consumidores individuales resultan beneficiados, toda vez que les resulta posible consumir más. En nuestro caso, el consumidor tipo 2 experimenta el mayor crecimiento. Cabe observar, que las funciones de reacción que se exponen en la Figura 4.4 corresponden al consumo total de los individuos, el cual está compuesto por el consumo tanto de bienes domésticos como extranjeros. En nuestro caso, la mayor parte del consumo del consumidor tipo 1 está destinado a bienes domésticos, en tanto que el consumidor 

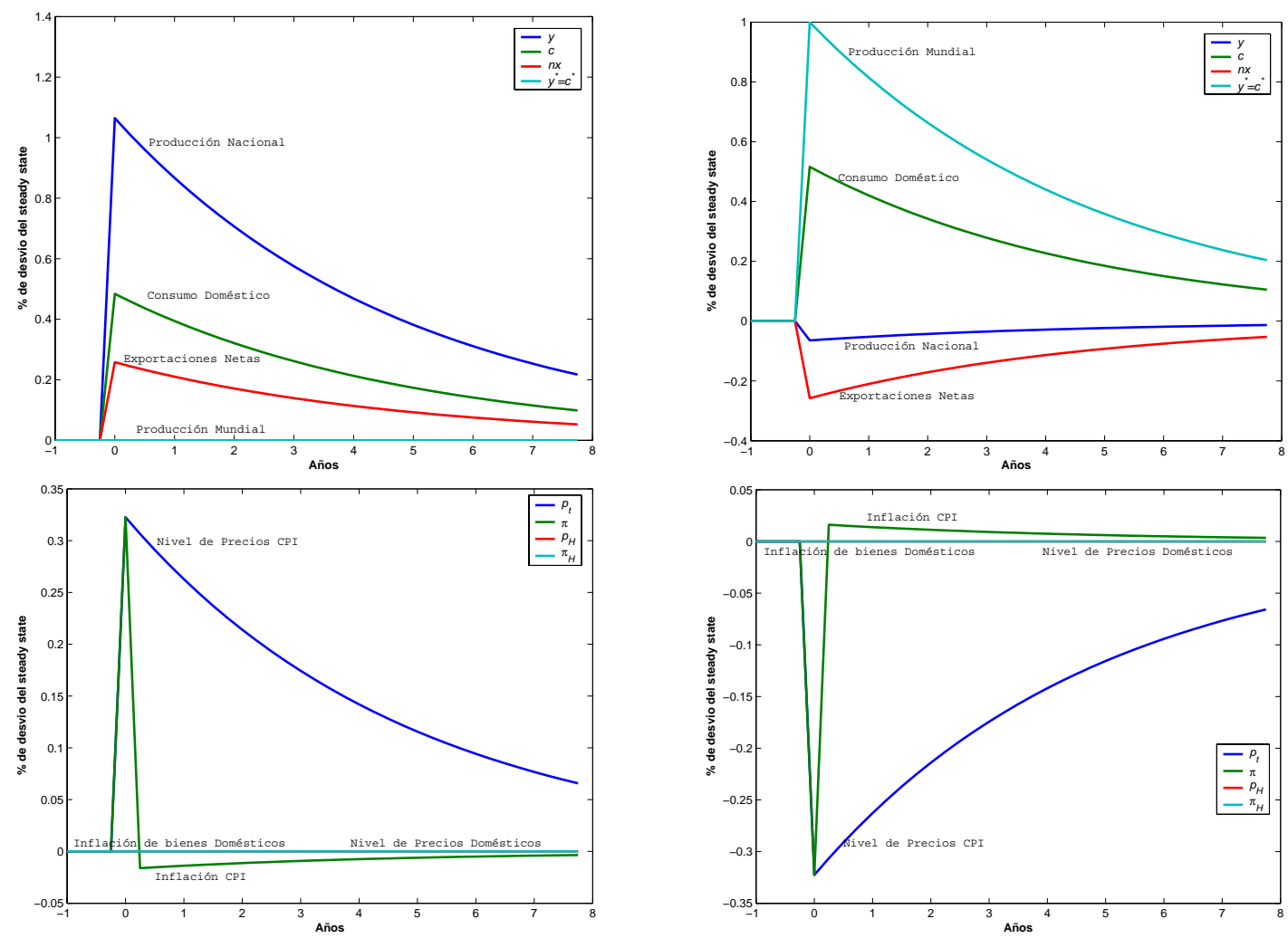

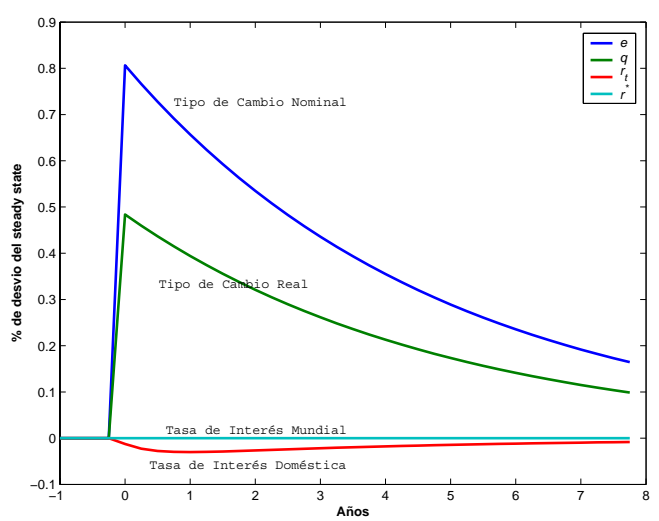

(a) Shock de Productividad Doméstica

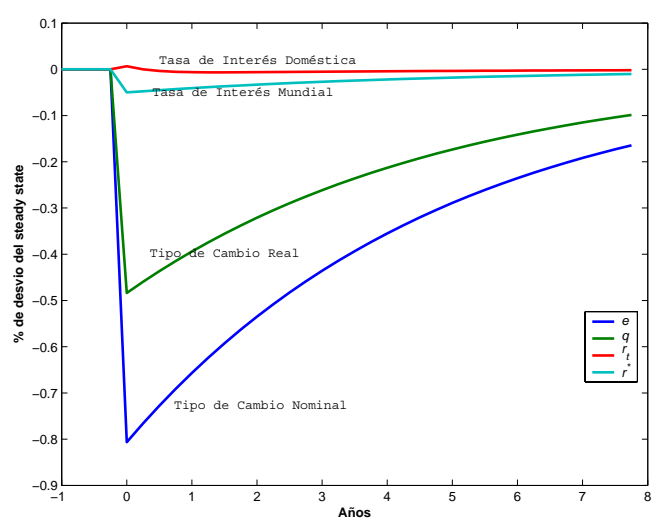

(b) Shock de Productividad Externa

Figura 4.1: Funciones de Reacción (Modelo OID) 

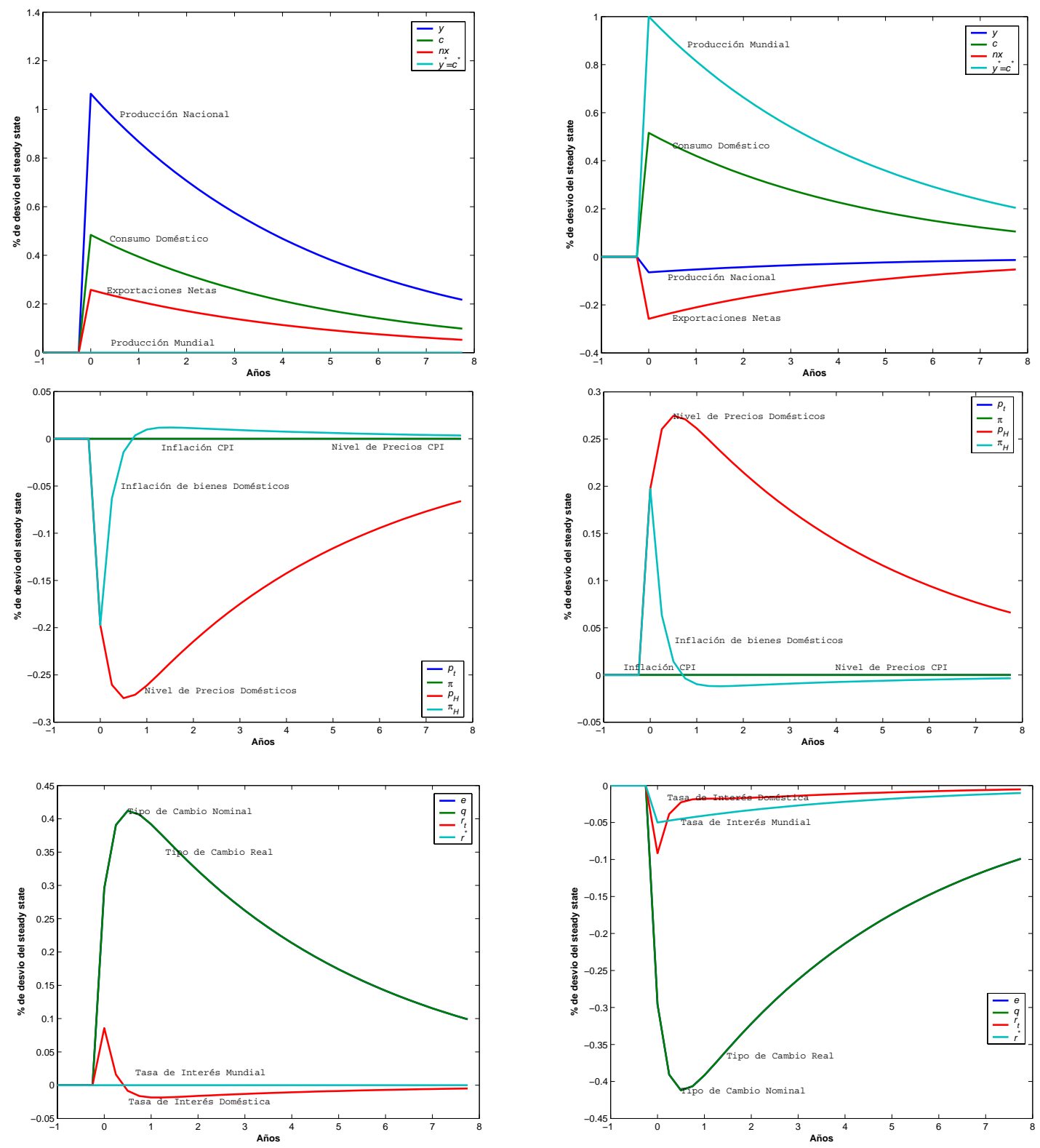

(a) Shock de Productividad Doméstica

(b) Shock de Productividad Externa

Figura 4.2: Funciones de Reacción (Modelo OI-IPC) 

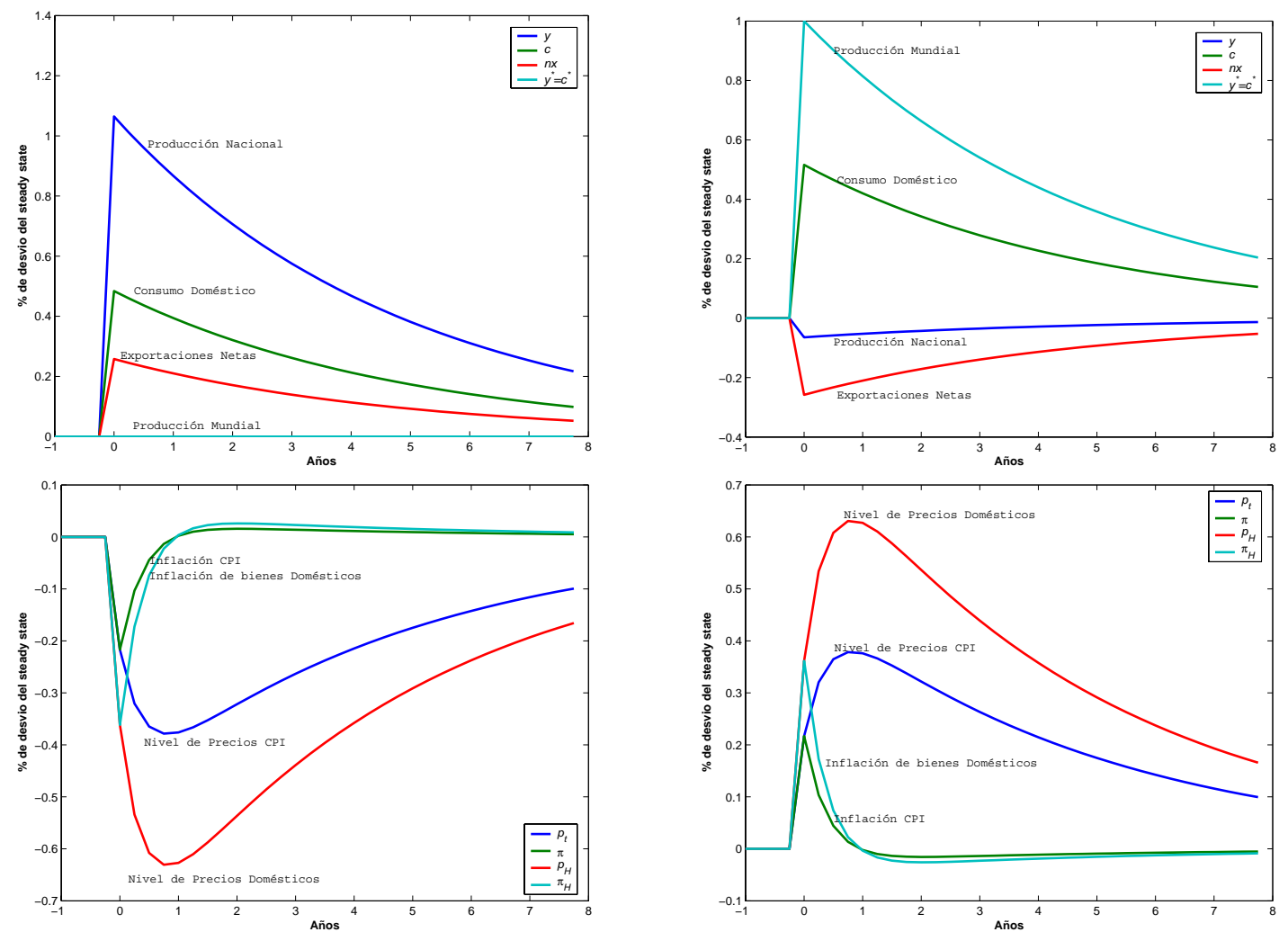

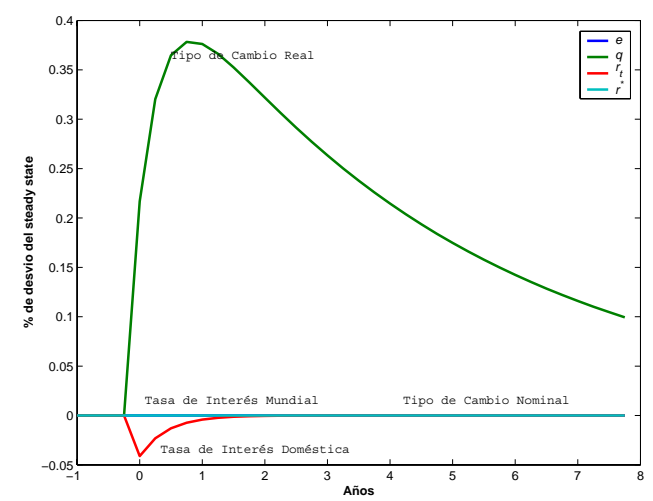

(a) Shock de Productividad Doméstica

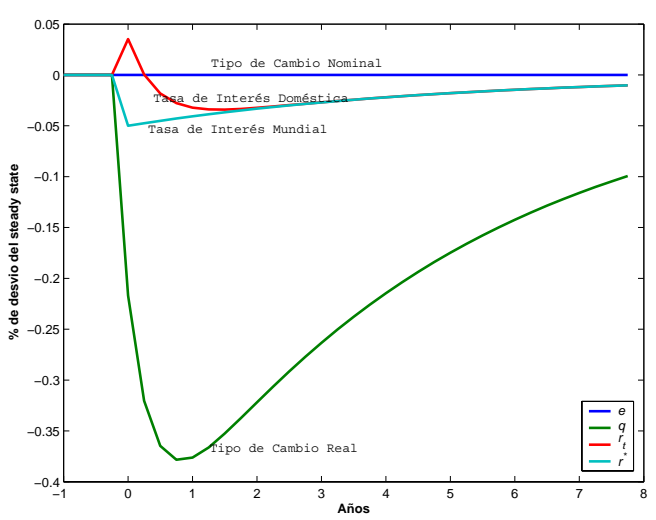

(b) Shock de Productividad Externa

Figura 4.3: Funciones de Reacción (Modelo OIF) 
tipo 2 destina un mayor porcentaje en bienes extranjeros que el consumidor tipo 1. Por lo tanto, la sumatoria de las funciones de reacción de los consumidores por su correspondiente proporción en el consumo nacional por la proporción de consumo de cada uno de bienes domésticos resulta igual a la reacción del consumo doméstico agregado.

\subsubsection{Shock de Productividad Externo}

Ante un shock de carácter externo, tanto la producción externa como el consumo externo, se incrementan en la misma medida. Por su parte, el consumo local, lo hace en menor medida que su grado de apertura comercial. Dado que el país pasa a importar más bienes, las exportaciones netas presentan valores negativos. Y dado que los bienes extranjeros resultan más baratos, la producción doméstica se reduce en un pequeño porcentaje. Por su parte, la autoridad monetaria mundial busca estabilizar la inflación mundial, éste decide reducir la tasa de interés mundial. Ahora bien, en la economía pequeña se consiguen resultados específicos dependiendo de la política monetaria adoptada.

\section{Objetivo de Inflación Doméstica - (OID)}

Bajo este contexto, tanto el nivel de precios IPC como la inflación caen agudamente al producirse el shock externo, debido a que la producción en el resto del mundo es más barata. A partir de allí, comienzan a subir. La inflación IPC lo hace de manera más abrupta, para volver a alcanzar su valor de steady state (esto es así, dado que las expectativas van ajustando su valor, en virtud de la $N K P C$ ), en tanto que el nivel de precios lo hace de manera más suave (ver Figura 4.1b) a efectos de poder lograr el objetivo de mantener fijo el nivel de precios domésticos. La tasa de interés doméstica prácticamente no se ve afectada por esta política. Como puede observarse, la inflación IPC, su nivel de precios, el tipo de cambio nominal y el real se comportan de manera opuesta considerando los shocks agregados domésticos y externos.

\section{Objetivo de Inflación $I P C-(O I-I P C)$}

Con una política $O I-I P C$, los precios de los bienes domésticos deben aumentar de modo considerable para poder compensar las importaciones más baratas. Esto se logra con un incremento del nivel de precios $p_{H}$, lo que a su vez produce una inflación de tipo $\pi_{H}$. Además, resulta necesario efectuar una reducción de la tasa de interés doméstica en la economía pequeña, según la Figura 4.2b.

\section{Fijación $P E G-O I F$}

En un sistema de carácter fijo, el tipo de cambio nominal se encuentra fijo, lo que obliga a la autoridad monetaria local a seguir (o imitar) la regla de la tasa de interés mundial. La reducción de la tasa de interés local ante la presencia de una contracción de la producción doméstica conlleva a una aumento de los precios en la economía pequeña, por lo que se produce un aumento de la tasa de inflación, tanto de precios domésticos como de carácter IPC (ver Figura 4.3b). 

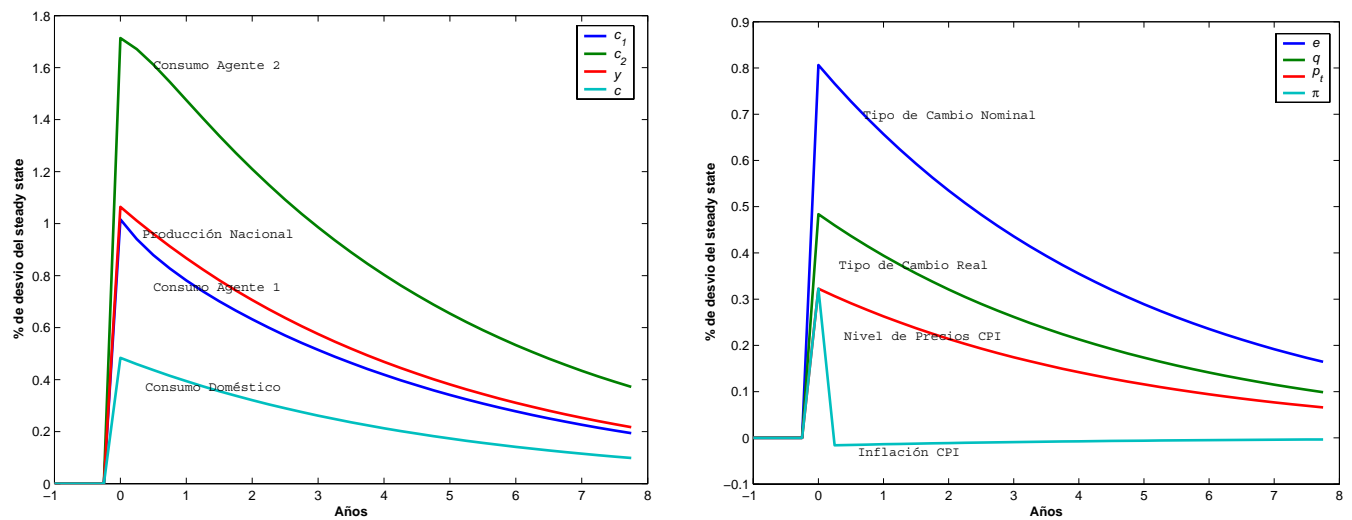

(a) Política OID.
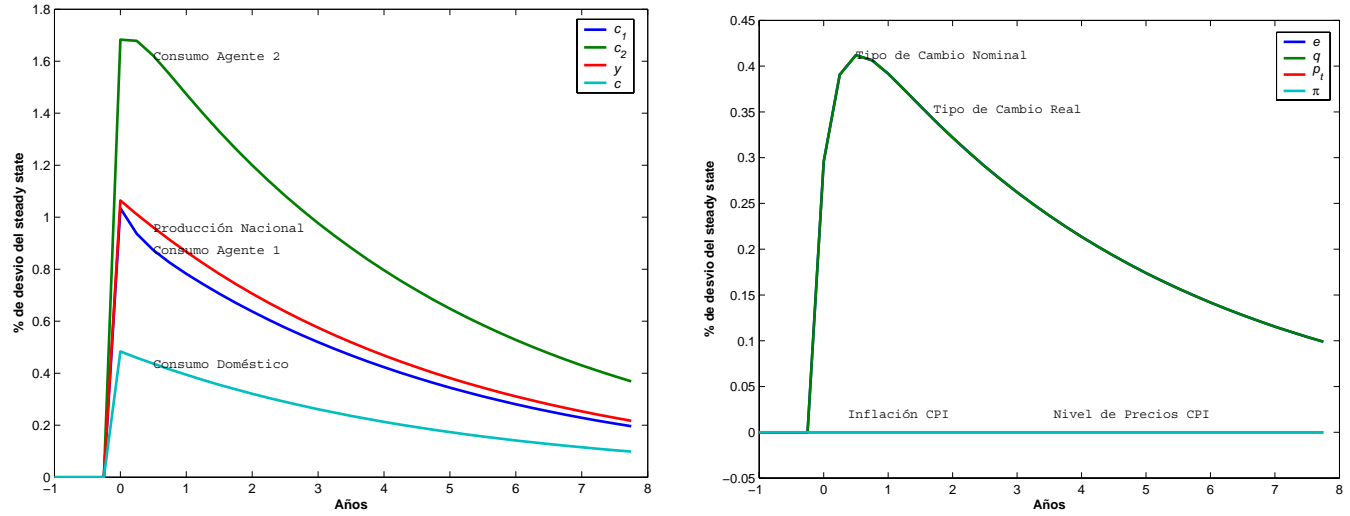

(b) Política OI-IPC.
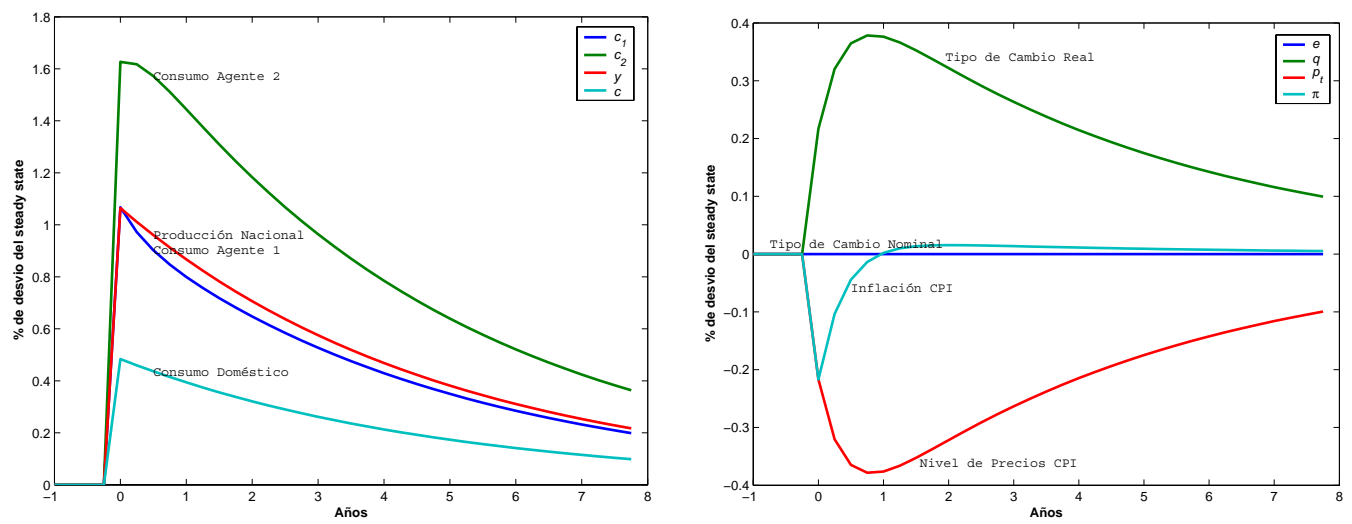

(a) Política OIF.

Figura 4.4: Situación de los Consumidores. Shock Agregado Nacional. 


\section{Situación de los Consumidores}

A diferencia del caso anterior, los consumidores reaccionan de manera casi similar, toda vez que se reduce el precio de los bienes extranjeros, y resulta más accesible adquirirlos, tanto para el consumidor tipo 2 (que ya destinaba una parte importante de su presupuesto a adquirirlos), como para el consumidor tipo 1, que ahora les resulta más barato que antes (ver Figura 4.5).

\subsection{Dinámica Distributiva}

En esta sección pasamos a analizar como se comporta el Coeficiente de Gini ante los diferentes shocks. En primer lugar si insertamos las ecuaciones (3.56) y (3.57) en la ecuación (3.58) y esta a su vez en la ecuación (3.59), resulta:

$$
\widehat{\operatorname{Gini} \widehat{\operatorname{Gini}}}=-\sum_{h} T_{h} \bar{£}_{h} \widehat{£}_{h, t}
$$

además, teniendo en cuenta que (3.56) contiene $\bar{£}_{h}$ y $\widehat{£}_{h, t}$, resulta posible analizar el cambio en el coeficiente de Gini ante variaciones de las distintas variables. De este modo, resulta:

$$
\overline{\text { Gini } \widehat{\text { Gini }}}=-\sum_{h} T_{h}\left(\frac{\overline{W k_{h}}}{\bar{P}}\left(\widehat{W}_{t}+\widehat{k}_{h, t}-\widehat{p}_{T}\right)+\bar{r} \overline{D_{h}}\left(\widehat{r}_{t}+\widehat{D}_{h, t}\right)-\overline{\overline{W N_{h}}} \widehat{\bar{P}}-\bar{D} \widehat{D}_{t}\right)
$$

De modo, que bajo el modelo planteado, el coeficiente de Gini se incrementará ante aumentos el nivel de precios $I P C$, o incrementos del salario/renta total superior en proporción al aumento del salario/renta de los sectores más bajos. Por su parte, un incremento del salario/renta que aumente los ingresos de los sectores más bajos en mayor medida que el resto de la población conlleva a una reducción de la desigualdad, toda vez que esto produce un aumento de los ingresos generales de la economía.

A partir del cuadro de situación expuesto al comienzo de este capítulo con respecto a los individuos que componen nuestra economía, resulta la Curva de Lorenz asociada (Figura 4.6). Las funciones de reacción del Coeficiente de Gini ante las tres políticas monetarias (OID, OI$I P C$ y $O I F)$ tanto para los shocks de carácter nacional como extranjero se presentan en la Figuras $4.7 \mathrm{a}$ y $4.7 \mathrm{~b}$ respectivamente).

Como primera observación, podemos destacar, que las funciones de reacción antes los shocks domésticos y externos resultan opuestos. Esto surge por el hecho que las variables de interés en nuestro modelo que conllevan implicancias distributivas $\left(r_{t}, \pi_{t}, p_{t}, \pi_{H}\right.$ y $\left.p_{H}\right)$ presentan un comportamiento opuesto como surge de lo comentado en la Sección 4.1.1. Estas conclusiones se encuentran expresadas de manera sintética en las Tablas 4.2 y 4.3 .

Bajo la especificación de este modelo, el coeficiente de Gini se reduce ante aumentos del salario real, lo que se produce con caídas del nivel de precios IPC. Ante shocks de carácter agregado domésticos, bajo la política monetaria $O I D$, el nivel de precios $p_{t}$ aumenta a efectos de mantener constante el nivel $p_{H}$, por tal motivo, la desigualdad sube en este modelo. 

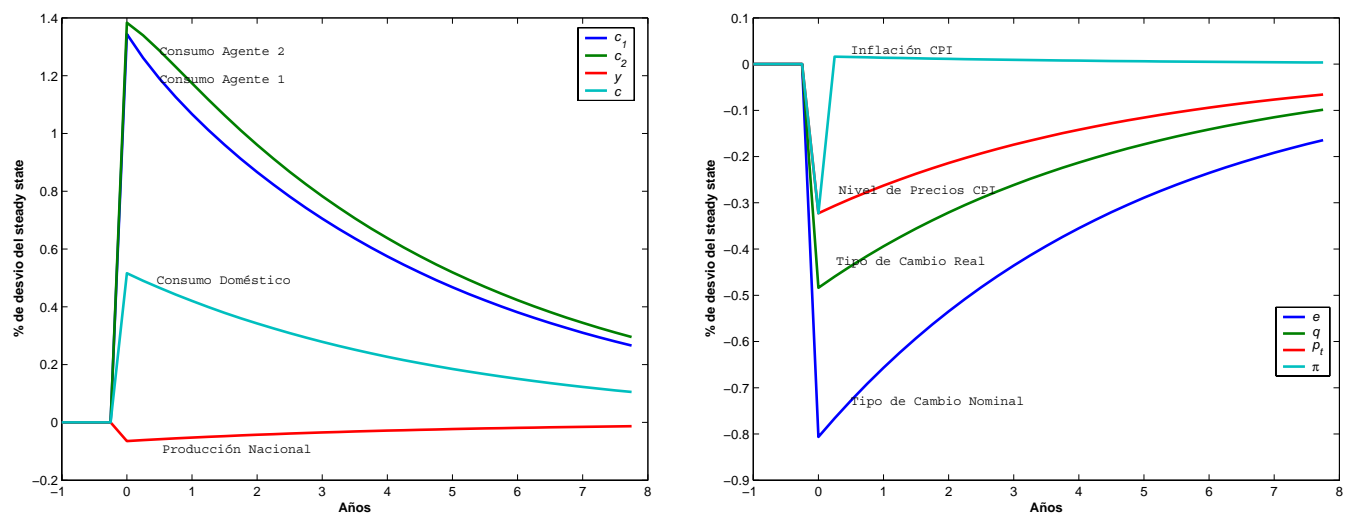

(a) Política OID.
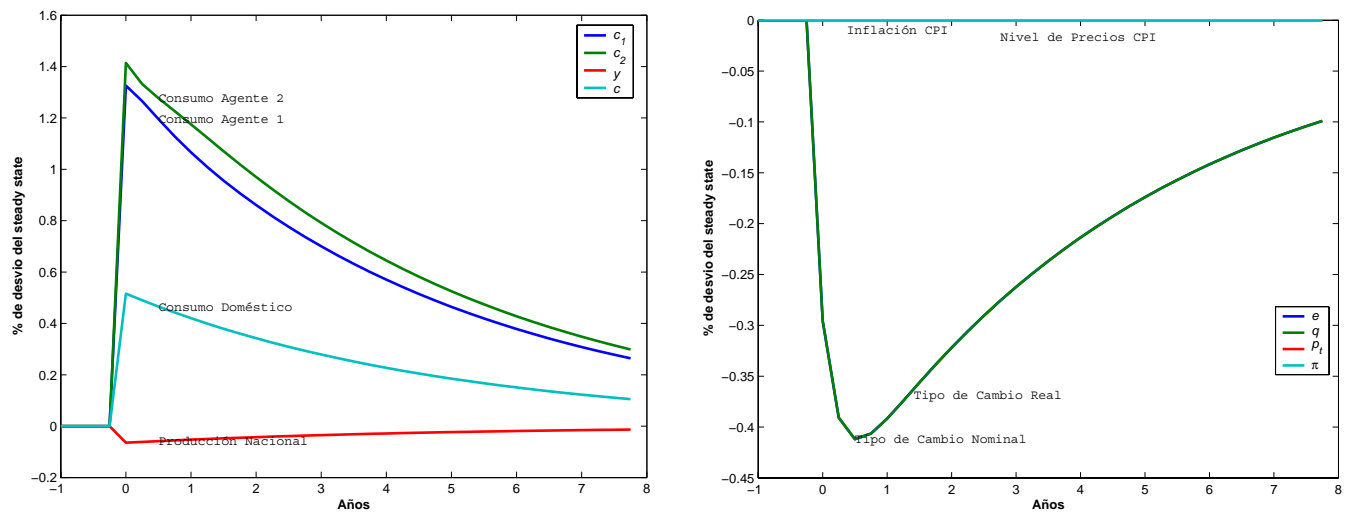

(b) Política OI-IPC.
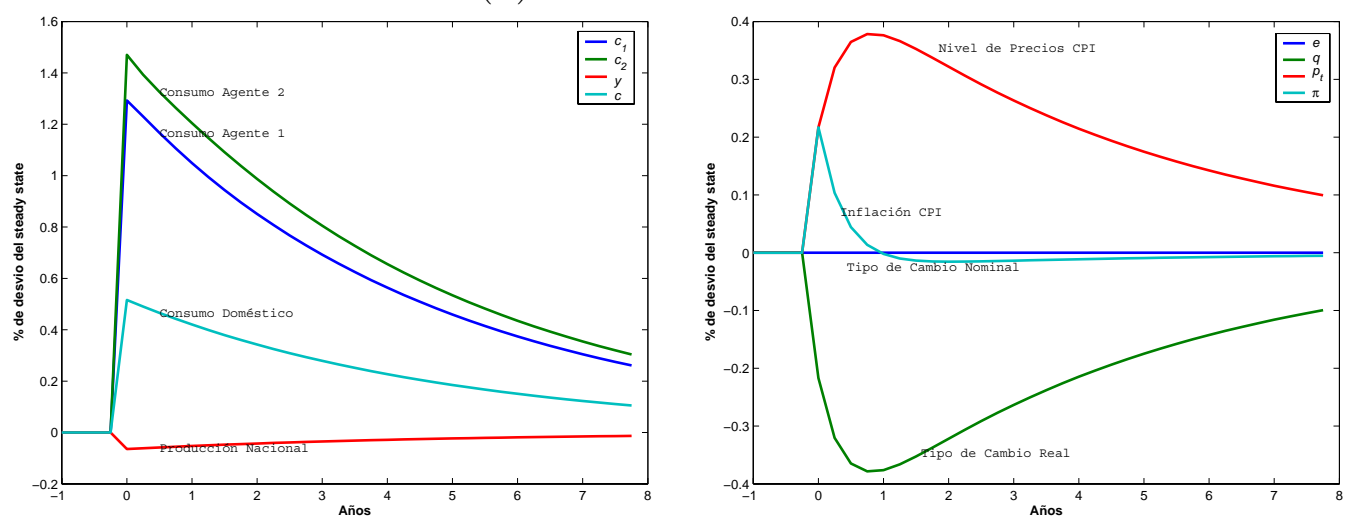

(c) Política OIF.

Figura 4.5: Situación de los Consumidores. Shock Agregado Externo. 
Asimismo, con esta política la reducción de la tasa de interés es mínima, por lo que los ingresos provenientes de rentas no varían en gran medida. Esto implica que la desigualdad no suba más de lo registrado.

Cuando la política es de carácter $O I-I P C$, la inflación $\pi_{t}$ es cero, por lo que los salarios reales aumentan, lo que conlleva a una reducción de la desigualdad. La tasa de interés experimenta un aumento al inicio, lo que contrarresta en un primer momento la reducción de la desigualdad. Posteriormente se reduce, por lo que los ingresos provenientes de rentas (que beneficia al sector 2 de la economía) se reducen, por lo que la desigualdad se reduce de manera mínima.

Por su parte, con la política es $O I F$, se registra la mayor caída en el nivel de precios, por tal motivo, el salario real de la economía experimenta el mayor crecimiento, que beneficia al sector 1 de la economía. Entonces, en este escenario se presenta la menor desigualdad registrada. A esto, cabe agregar un caída minima en la tasa de interés, lo cual reduce el ingreso del sector 2 (que es el que posee una mayor participación de los activos financieros de la economía).

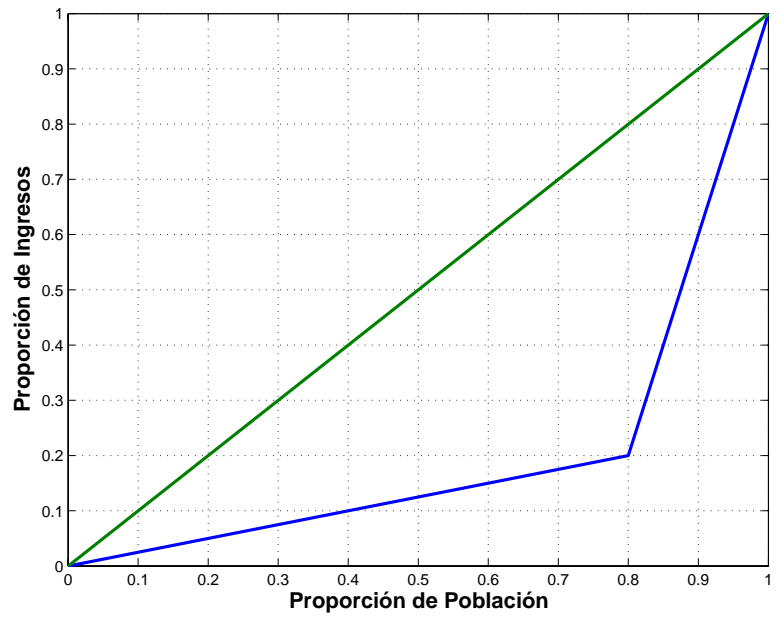

Figura 4.6: Curva de Lorenz (2 Consumidores).

Cuando el shock es de carácter externo, se producen los resultados opuestos con respecto a las variables $p_{t}, \pi_{t}, p_{H}$ y $\pi_{H}$ en comparación con el shock de carácter nacional. Por tal motivo, con una política de tipo $O I D$, se registra la menor desigualdad, en tanto que ahora bajo la política $O I F$, se registra la mayor desigualdad. Nuevamente, bajo la política $O I-I P C$, la desigualdad registra cambios mínimos.

Este ejercicio puede replicarse para un caso real y ver de que manera varía el coeficiente de Gini. Por tal motivo, utilizamos datos de la Encuesta Permanente de Hogares para el año 1998. Así, definimos a 5 individuos, que representan cada uno de los quintiles poblacionales. El indiviuo 1 (o quintil 1) recibe el 3,51\% del ingreso nacional. El 2, el 8,52\%. El 3, el 13,43\%. El 4, el 21,75\%. Y el 5, el más rico de esta economía, el 52,77\%. La curva de Lorenz que caracteriza a esta economía, se representa en la Figura 4.8.

En este caso, puede observarse que los sectores más bajos resultan ser los más vulnerables, dado que su participación en el ingreso nacional es muy baja, recién el individuo 4 recibe una 


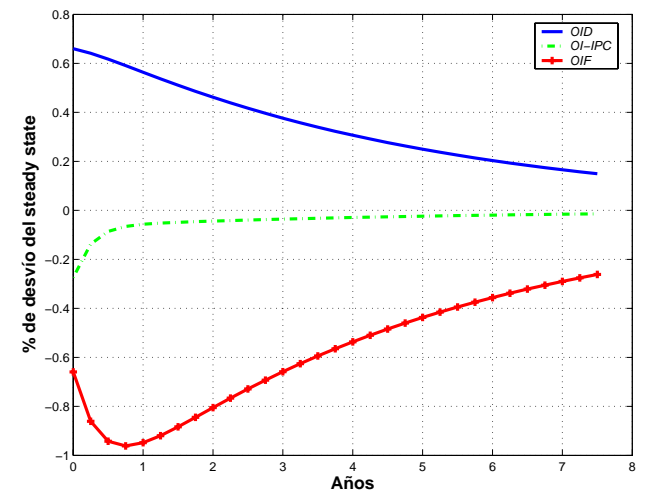

(a) Shock de Productividad Doméstica

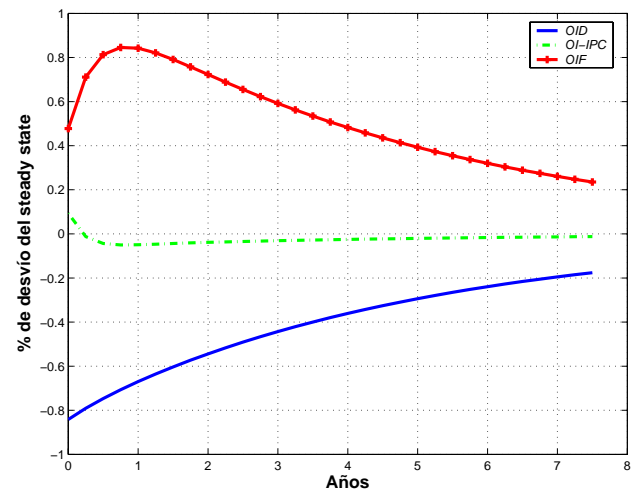

(b) Shock de Productividad Externa

Figura 4.7: Funciones de Reacción del Coeficiente de Gini con 2 consumidores.

\section{Cuadro 4.2: Relaciones (Shock Doméstico Agregado)}

\begin{tabular}{llllllllllllll}
\hline \hline Modelo & $y$ & $c$ & $n x$ & $y^{*}$ & $e$ & $q$ & $r$ & $r^{*}$ & $p_{t}$ & $p_{h}$ & $\pi_{t}$ & $\pi_{h}$ & Gini \\
\hline OID & $\uparrow$ & $\uparrow$ & $\uparrow$ & $\longrightarrow$ & $\uparrow$ & $\uparrow$ & $\downarrow$ & $\longrightarrow$ & $\uparrow$ & $\longrightarrow$ & $\uparrow$ & $\longrightarrow$ & $\uparrow$ \\
OI-IPC & $\uparrow$ & $\uparrow$ & $\uparrow$ & $\longrightarrow$ & $\uparrow$ & $\uparrow$ & $\uparrow \downarrow$ & $\longrightarrow$ & $\longrightarrow$ & $\downarrow$ & $\longrightarrow$ & $\downarrow$ & $\downarrow \longrightarrow$ \\
OIF & $\uparrow$ & $\uparrow$ & $\uparrow$ & $\longrightarrow$ & $\longrightarrow$ & $\uparrow$ & $\longrightarrow$ & $\longrightarrow$ & $\downarrow$ & $\downarrow$ & $\downarrow$ & $\downarrow$ & $\downarrow$ \\
\hline \hline
\end{tabular}

participación casi igual a su porcentaje poblacional (representa el $20 \%$ de la población y recibe el $21,75 \%)$.

En la Figura 4.9 se grafican las funciones de reacción para los shocks agregados de esta economía. El comportamiento de las funciones resulta similar al del caso con 2 consumidores. Sin embargo, puede observarse que las curvas se encuentran más separadas entre sí. Este hecho se debe, a que la caracterización de esta economía es un tanto más extrema que el caso anterior. Aquí, los 3 primeros individuos reciben una participación muy baja del ingreso nacional (el $25,47 \%$ ), lo que los vuelve muy vulnerables a los diferentes shocks. Cuando el shock es agregado nacional, con una política $O I D$, se experimenta la mayor inflación de precios $I P C$, por tal motivo, el salario real experimenta la mayor caída. Caso contrario ocurre con la política de fijación $P E G$, que en este caso experimenta el mayor aumento, y por ende beneficia principalmente a los 4 primeros quintiles. Como se explicó en la sección anterior, cuando el shock es de carácter externo, las funciones de reacción se comportan de manera opuesta al caso de un shock de

Cuadro 4.3: Relaciones (Shock Internacional Agregado)

\begin{tabular}{llllllllllllll}
\hline \hline Modelo & $y$ & $c$ & $n x$ & $y^{*}$ & $e$ & $q$ & $r$ & $r^{*}$ & $p_{t}$ & $p_{h}$ & $\pi_{t}$ & $\pi_{h}$ & Gini \\
\hline OID & $\downarrow$ & $\uparrow$ & $\downarrow$ & $\uparrow$ & $\downarrow$ & $\downarrow$ & $\longrightarrow$ & $\downarrow$ & $\downarrow$ & $\longrightarrow$ & $\downarrow \uparrow$ & $\longrightarrow$ & $\downarrow \longrightarrow$ \\
OI-IPC & $\downarrow$ & $\uparrow$ & $\downarrow$ & $\uparrow$ & $\downarrow$ & $\downarrow$ & $\downarrow$ & $\downarrow$ & $\longrightarrow$ & $\uparrow$ & $\longrightarrow$ & $\uparrow \downarrow \longrightarrow$ & $\uparrow \downarrow \longrightarrow$ \\
OIF & $\downarrow$ & $\uparrow$ & $\downarrow$ & $\uparrow$ & $\longrightarrow$ & $\downarrow$ & $\downarrow$ & $\downarrow$ & $\uparrow$ & $\uparrow$ & $\uparrow \downarrow \longrightarrow$ & $\uparrow \downarrow \longrightarrow$ & $\uparrow \downarrow \longrightarrow$ \\
\hline \hline
\end{tabular}




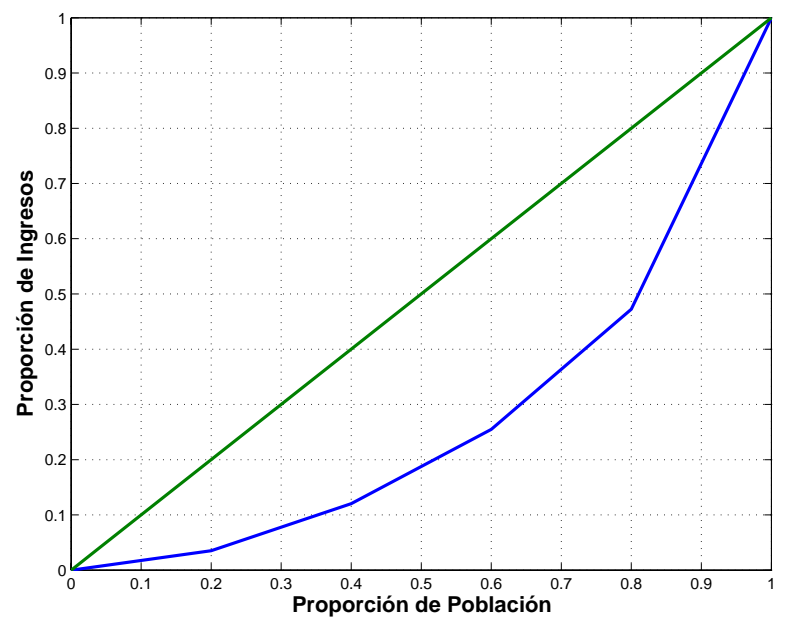

Figura 4.8: Curva de Lorenz (5 Consumidores).

carácter nacional, por lo que se vuelven a tener los mismos resultados en términos cualitativos (el crecimiento del Gini es mayor con política $O I F$, y cae con la política $O I D$ ), pero mayores en términos absolutos.

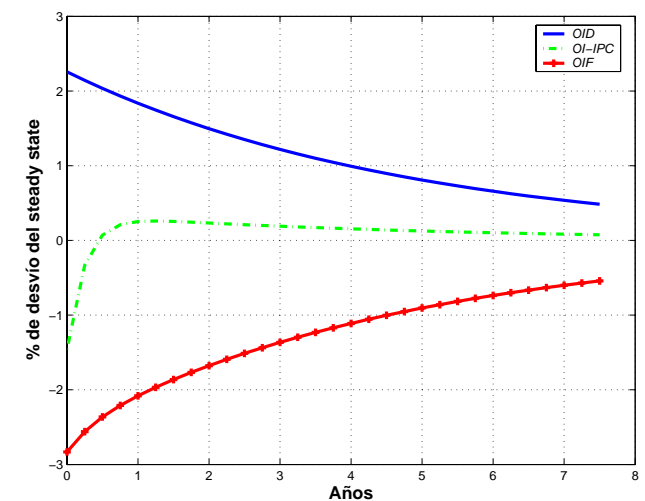

(a) Shock de Productividad Doméstica

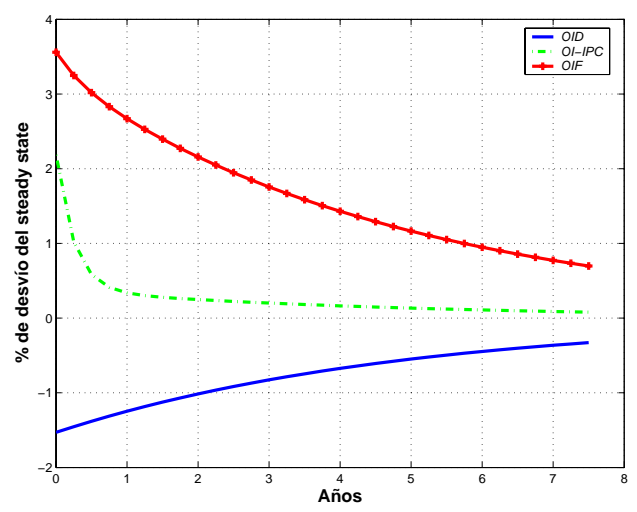

(b) Shock de Productividad Externa

Figura 4.9: Funciones de Reacción del Coeficiente de Gini con 5 consumidores.

\subsubsection{Shock Individual}

\section{Shock de Productividad del individuo $i$}

Siguiendo a Krussel y Smith, nuestro modelo plantea la posibilidad de que se produzca un shock de productividad de carácter individual, o sea que afecta únicamente a un determinado sector de la población. En nuestro caso base, de 2 tipos de consumidores, planteamos la situación, en el que el shock afecta al consumidor tipo 1 y tipo 2 (Figuras 4.10a y 4.10b respectivamente). 


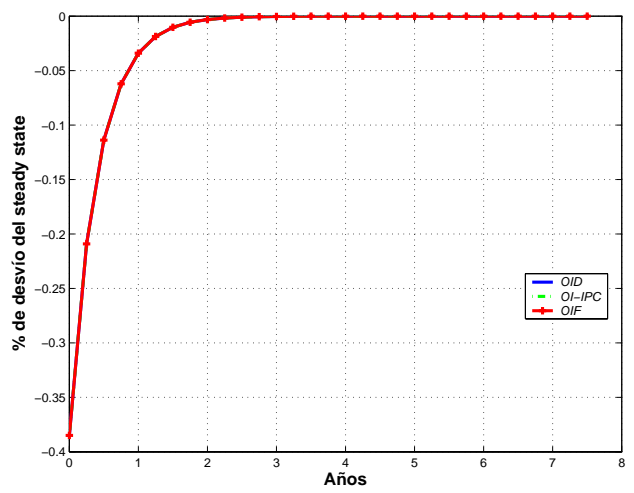

(a) Shock del Individuo 1

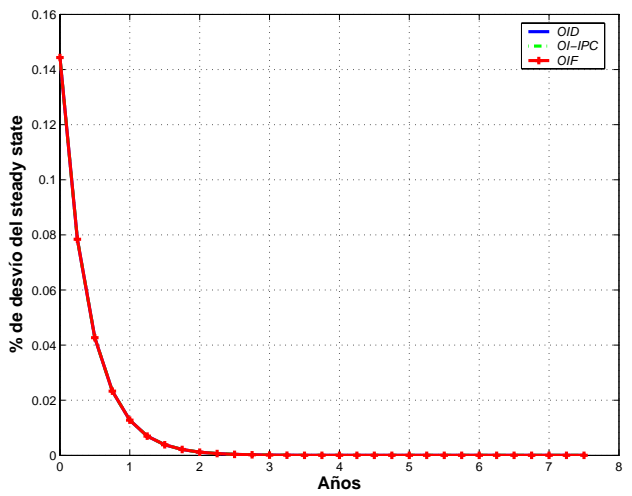

(b) Shock del Individuo 2

Figura 4.10: Análisis del Coeficiente de Gini ante shock Individual (2 consumidores).

En este caso, todo shock de productividad del individuo $i$ conlleva a un incremento de sus ingresos, a partir del hecho de que le resulta posible obtener una mayor renta por la misma cantidad de trabajo aportado. Si este individuo pertenece al sector con menores ingresos, la desigualdad se reduce, en tanto que aumenta en el caso opuesto. Como puede apreciarse, ante un shock de producción del individuo tipo 1, que es el de menor ingreso (Figura 4.10a), la desigualdad cae. En tanto que si el shock afecta solamente al individuo 2 (que es el de mayores ingresos), la desigualdad aumenta (Figura 4.10b).

En un escenario en el que existen 5 consumidores, donde el perteneciente al sector 5 es el que recibe mayores ingresos, un shock individual que beneficie a cualquiera de los 4 primeros sectores, implica una reducción de la desigualdad. En tanto que si el shock afecta al individuo del ultimo sector, la desigualdad aumenta (Figura 4.11)

Otra relación importante que surge del modelo planteado, es que cuando el shock de carácter individual influye en los sectores que reciben menos ingresos, la desigualdad se reduce más considerablemente que si ocurre en los sectores medios o superiores. Cabe aclarar, que estos shocks individuales no resultan ser tan importantes como para afectar las variables de inflación o términos de intercambio en la economía pequeña, por lo que la reacción del coeficiente de Gini se comporta de manera similar bajo las tres políticas monetarias planteadas (OID, OI-IPC y $O I F)$.

\section{El Carácter Antagónico del Shock de Productividad del individuo $i$}

El modelo plantea la situación en el un shock de productividad que afecta al individuo $i$ le genera la posibilidad de poder consumir más bienes, sin embargo, el resto de los individuos se ven influenciados de manera negativa, ya que se ve reducida su capacidad de adquirir bienes, a partir del hecho de que el ingreso del sector beneficiado se incrementa en mayor medida que la producción de bienes. Por tal motivo, un shock de productividad del individuo $i$, incrementa el consumo de $i$ y reduce el del agente $j$, para todo $i \neq j$. esto se refleja en la Figura 4.12, que plantea el caso para 5 consumidores. 


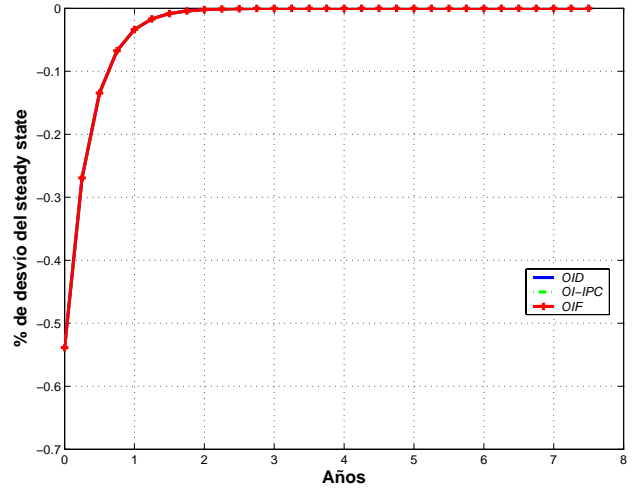

(a) Shock del Individuo 1

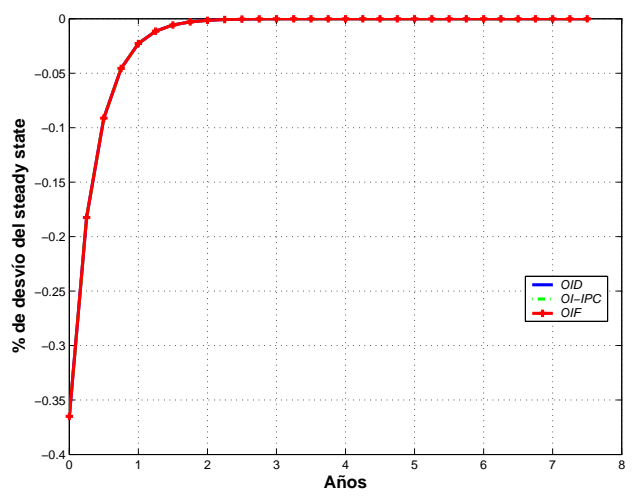

(c) Shock del Individuo 3

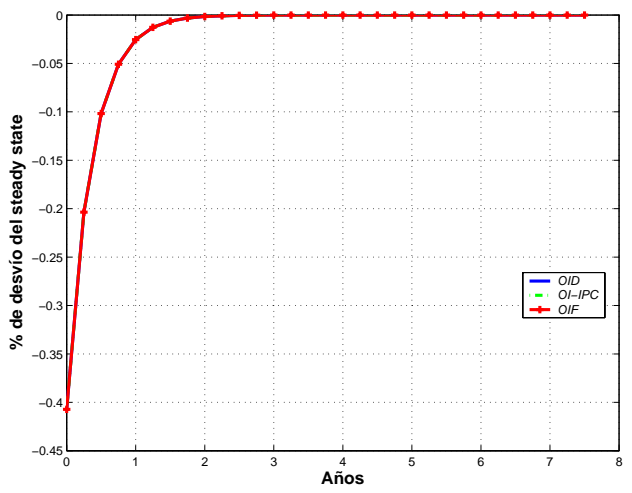

(b) Shock del Individuo 2

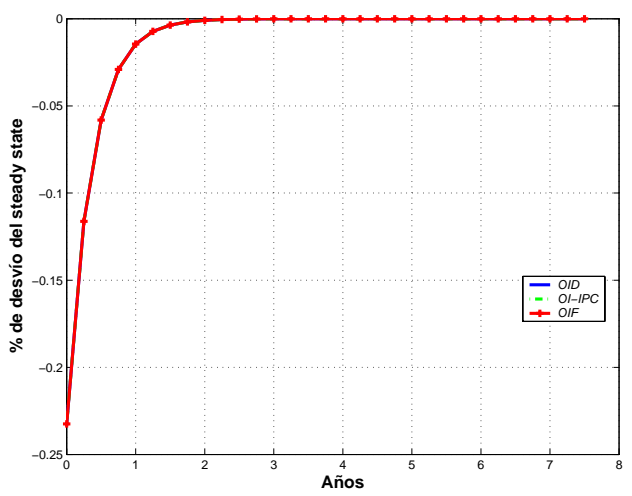

(d) Shock del Individuo 4

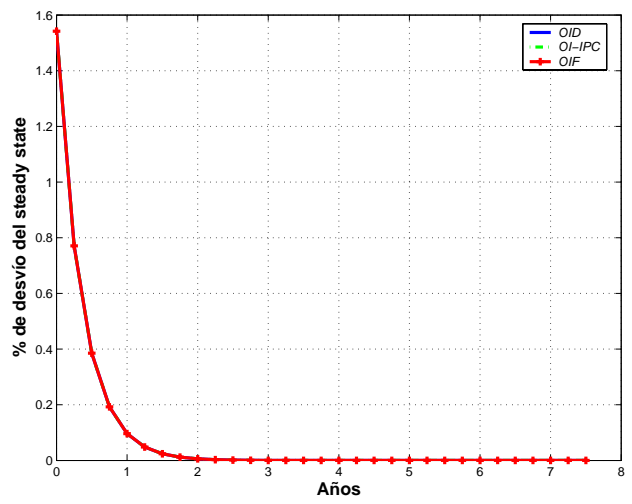

(e) Shock del Individuo 5

Figura 4.11: Análisis del Coeficiente de Gini ante shock Individual (5 consumidores) 


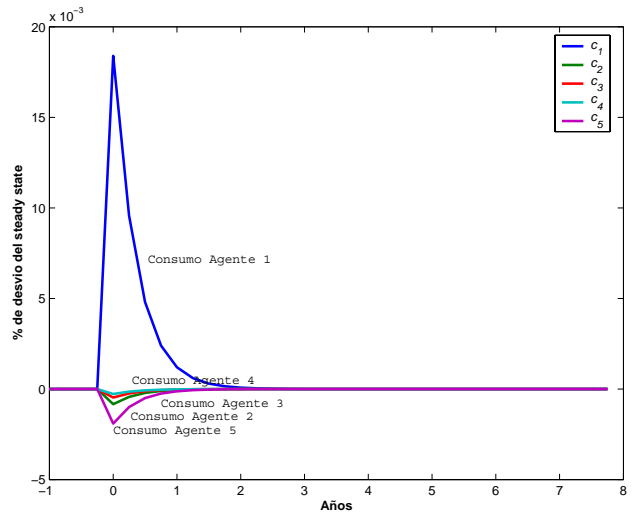

(a) Shock del Individuo 1

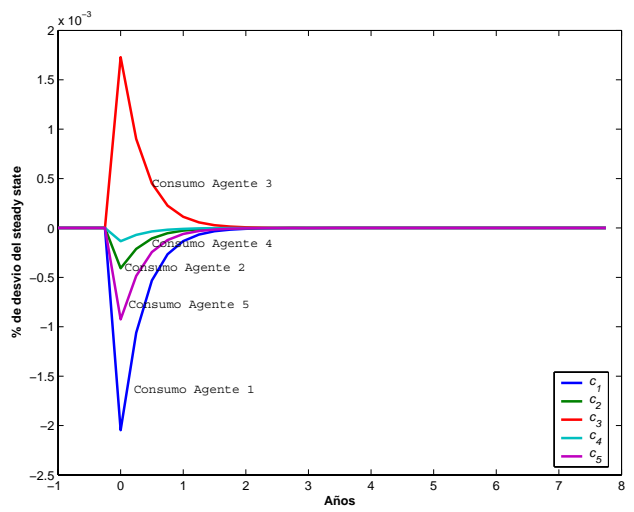

(c) Shock del Individuo 3

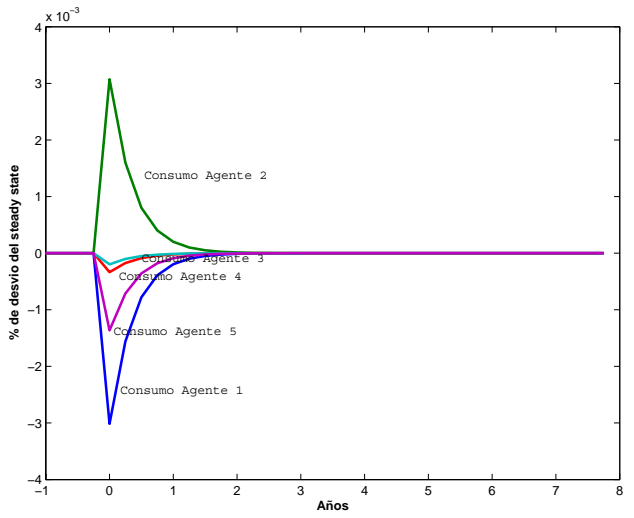

(b) Shock del Individuo 2

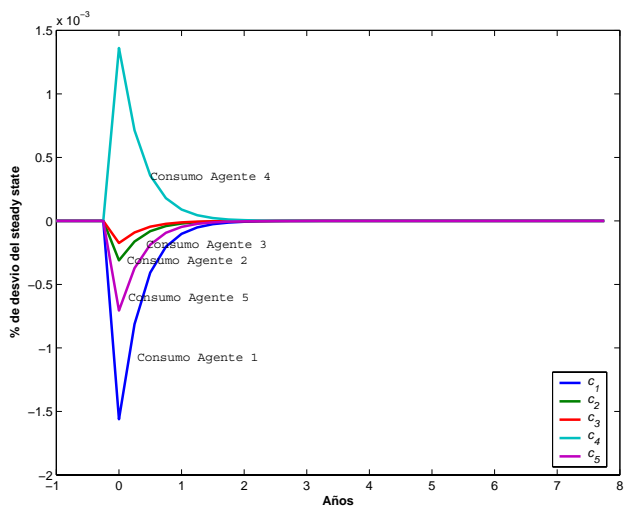

(d) Shock del Individuo 4

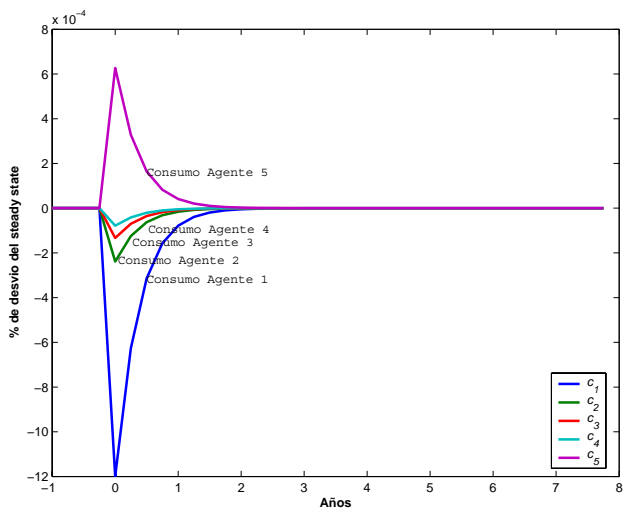

(e) Shock del Individuo 5

Figura 4.12: Consumo Individual (5 consumidores) 


\section{Capítulo 5}

\section{Conclusiones}

Este trabajo intentó presentar una mirada diferente al análisis de la distribución del ingreso. En general, la macroeconomía tiende al estudio de las variables agregadas de una economía. Sin embargo, la implementación de diferentes agentes heterogéneos (que componen el agregado nacional) brinda la posibilidad de extraer conclusiones para cada sector en particular. Así, resulta posible analizar diferentes categorías de consumidores que difieren en sus habilidades, dotaciones, expectativas, etc. En tanto que también resulta posible analizar desde el lado de la producción (imaginemos una economía con muchos sectores, ya sea de bienes primarios, intermedios y finales, o importadoras y productoras de bienes y servicios por citar unos ejemplos). Este trabajo, se desarrolló dentro de la teoría del equilibrio general dinámico estocástico, en el que una economía abierta pequeña, influenciada por el resto del mundo, donde su productividad se ve afectada por shocks de carácter agregado tanto doméstico como externo. Dicha economía (compuesta por agentes heterogéneos), también se ve afectada por shocks de tipo individual que influyen a un sector específico de la población. A tal efecto, se analizó de qué forma, las diferentes políticas monetarias implementadas en dicha economía, modifican la distribución del ingreso, llegándose a las siguientes conclusiones:

- un shock de carácter agregado nacional beneficia la distribución del ingreso toda vez que se reduzca el nivel de precios $I P C$, esto es cuando la política es de carácter $P E G$ o de Inflación Fija $I P C$,

- la política de tipo OID, es la que brinda los resultados más disímiles, ya que ante un shock doméstico produce mayor desigualdad y con un shock externo, la desigualdad se reduce drásticamente,

- el shock de carácter externo brinda resultados opuestos a los del de carácter nacional agregado,

- en ambos casos, el consumo individual se incrementa, ya sea por aumento del ingreso (shock nacional), o abaratamiento de los bienes (shock externo),

- un shock de tipo individual reduce la desigualdad toda vez que involucre a los sectores de menores ingresos, en tanto que la aumenta si afecta a los sectores con mayores ingresos, 
- cuando se produce un shock que afecta la productividad del individuo $i$, su consumo se incrementa, en tanto que el del resto de los sectores se reduce.

Como se estableció al inicio de esta sección, el comprobar la influencia de la política monetaria en la desigualdad no resulta tradicionalmente investigado desde esta óptica, por lo que se recurrió al empleo de un modelo de tipo Neo Keynesiano para poder llevarlo a cabo. Sin embargo, dado que constituyó un primer paso para el análisis, existen muchos caminos de acción futuros, cómo ser:

- incorporar rigideces de salario,

- ampliar el modelo de modo tal que se incluya al capital como segunda fuente de producción (tal como lo hacen Christiano y otros),

- considerar que la función de producción incorpore bienes importados, de modo de observar como afectan las variaciones de precios externos en el consumo local,

- si se incorpora el capital en la restricción presupuestaria de los individuos, se podrá analizar además la desigualdad de la riqueza de la economía,

- la inclusión del gobierno ya sea como consumidor de bienes, agente que recauda impuestos y brinda transferencias, así como la autoridad que se ocupa de la administrar la oferta monetaria, brinda la posibilidad de enriquecer el modelo y extraer conclusiones adicionales.

Por último, y ya desde una perspectiva totalmente diferente, poder comprobar de manera empírica mediante métodos de estimación $V A R$ si estas conclusiones se han observado en la realidad en una economía de estas características constituye una alternativa posible para poder llevarlo a cabo en futuras investigaciones. 


\section{Bibliografía}

[1] S. Albanessi, Inflation and inequality, Working Paper, New Yok University (2002).

[2] Satyajit Chatterjee, Transitional dynamics and the distribution of wealth in a neoclassical growth model, Journal of Public Economics 54 (1994), no. 1, 97-119.

[3] Frank Cowell, Measurement of inequality, Handbook of Income Distribution, vol. 1, Elsevier, 6 2000, pp. 87-166.

[4] Huffman y Wynne Dolmas, Inequality, inflation and central bank independence, Canadian Journal of Economics. (2000).

[5] Haliassos y Japelli Guiso, Household portfolios, Cambridge, MA. MIT Press. (2002).

[6] Jose-Victor Rios-Rull, Working in the market, working at home, and the acquisition of skills: A general-equilibrium approach, American Economic Review 83 (1993), no. 4, 893907.

[7] Harald Uhlig, A toolkit for analysing nonlinear dynamic stochastic models easily, QM \& RBC Codes, Quantitative Macroeconomics \& Real Business Cycles (1998).

[8] Burkhard Heer y Alfred Maußner, Dynamic general equilibrium modelling, Tech. report, 2005.

[9] Abhijit Banerjee y AndrewÑewman, Occupational choice and the process of development, Discussion Papers 911, Northwestern University, Center for Mathematical Studies in Economics and Management Science, Jul 1990.

[10] Per Krusell y Anthony Smith, Income and wealth heterogeneity in the macroeconomy, Journal of Political Economy 106 (1998), no. 5, 867-896.

[11] _ Income and wealth heterogeneity, portfolio choice, and equilibrium asset returns, GSIA Working Papers 1997-45, Carnegie Mellon University, Tepper School of Business, undated.

[12] Thomas Cooley y Edward Prescott, Economic growth and business cycles, en Frontiers of Business Cycle Research, editedo por Thomas F. Cooley, Princeton, Princeton University Press. (1995). 
[13] David Backus; Patrick Kehoe y Finn Kydland, International business cycles: Theory and evidence, en Frontiers of Business Cycle Research, editedo por Thomas F. Cooley, Princeton, Princeton University Press. (1995).

[14] Ana Castañeda; Javier Diaz-Gimenez y Jose-Victor Rios-Rull, Exploring the income distribution business cycle dynamics, Journal of Monetary Economics 42 (1998), no. 1, 93-130.

[15] Oded Galor y Joseph Zeira, Income distribution and macroeconomics, Review of Economic Studies 60 (1993), no. 1, 35-52.

[16] Stephanie Schmitt-Grohé y Martín Uribe, Optimal and monetary policy under sticky prices, mimeo. (2001).

[17] Julio Rotemberg y Michael Woodford, Dynamic general equilibrium models with imperfectly competitive markets, en Frontiers of Business Cycle Research, editedo por Thomas F. Cooley, Princeton, Princeton University Press. (1995).

[18] Jordi Gali y Tommaso Monacelli, Optimal monetary policy and exchange rate volatility in a small open economy, Boston College Working Papers in Economics 438, Boston College Department of Economics, Jan 1999.

[19] Caselli y Ventura, A representative consumer theory of distribution, Working papers 96-11, Massachusetts Institute of Technology (MIT), Department of Economics, 1996. 


\section{Apéndice A}

\section{Comprobaciones Matemáticas}

\section{A.1. Fijación de precios óptimos en el modelo de Calvo}

A partir de Calvo (1983) se supone que cada firma individual firm fija sus precios con probabilidad $1-\theta$ cada período, independientemente del tiempo que pasó desde el último ajuste de precios. Así, cada período la medida $1-\theta$ de firmas aleatoriamente seleccionadas fijan sus precios, mientras que la fracción $\theta$ mantienen sus precios invariantes. Dado $P_{H, t+k} \mathrm{y}$ $\bar{P}_{H, t}$, bajo la estructura de Calvo, $P_{H, t+k}=P_{H, t}$ con probabilidad $\theta^{k}$ para $k=0,1,2, \ldots$. Dado que todas las firmas establecen los precios en algún momento, se establecerá el mismo precio y por lo tanto, se descarta el subíndice $j$.

Cuando se establece un nuevo precio en el período $t$, la firma $j$ busca maximizar el valor corriente de sus dividendos, condicional en que el precio resulte efectivo:

$$
\begin{aligned}
& \operatorname{máx}_{\bar{P}_{H, t}} \sum_{k=0}^{\infty} \theta^{k} E_{t}\left\{Q_{t, t+k}\left[Y_{t+k}\left(\bar{P}_{H, t}-M C_{t+k}^{n}\right)\right]\right\} \\
& \text { s.t. } \quad Y_{t+k} \leq\left(\frac{\bar{P}_{H, t}}{P_{H, t+k}}\right)^{-\varepsilon}\left(C_{H, t+k}+\int_{0}^{1} C_{H, t+k}^{i} d i\right)=Y_{t+k}^{d}\left(\bar{P}_{H, t}\right)
\end{aligned}
$$

donde $\left.M C_{t}^{n}\right)=\frac{(1-\tau) W_{t}}{A_{t}}$ denota el costo nominal marginal. Así, $\bar{P}_{H, t}$ debe satisfacer la condición de primer orden:

$$
\left.\sum_{k=0}^{\infty} \theta^{k} E_{t}\left\{Q_{t, t+k} Y_{t+k}\left(\bar{P}_{H, t}-\frac{\varepsilon}{\varepsilon-1} M C_{t+k}^{n}\right)\right)\right\}=0
$$

Utilizando la relación $Q_{t, t+k}=\beta^{k}\left(\frac{C_{t+k}}{C_{t}}\right)^{-\sigma}\left(\frac{p_{t}}{P_{t+k}}\right)$, se puede reexpresar la condición anterior como

$$
\sum_{k=0}^{\infty}(\beta \theta)^{k} E_{t}\left\{P_{t+k}^{-1} C_{t+k}^{-\sigma} Y_{t+k}\left(\bar{P}_{H, t}-\frac{\varepsilon}{\varepsilon-1} M C_{t+k}^{n}\right)\right\}
$$

o, en términos de variables estacionarias,

$$
\sum_{k=0}^{\infty}(\beta \theta)^{k} E_{t}\left\{C_{t+k}^{-\sigma} Y_{t+k} \frac{P_{H, t-1}}{P_{t+k}}\left(\frac{\bar{P}_{H, t}}{P_{H, t-1}}-\frac{\varepsilon}{\varepsilon-1} \Pi_{t-1, t+k}^{H} M C_{t+k}\right)\right\}=0
$$


donde $\Pi_{t-1, t+k}^{H} \equiv \frac{P_{H, t+k}}{P_{H, t-1}}$, y $M C_{t+k}=\frac{M C_{t+k}^{n}}{P_{H, t+k}}$. Log-linealizando la condición previa en el steadystate obtiene

$$
\bar{P}_{H, t}=P_{H, t-1}+\sum_{k=0}^{\infty}(\beta \theta)^{k} E_{t}\left\{\pi_{H, t+k}\right\}+(1-\beta \theta) \sum_{k=0}^{\infty}(\beta \theta)^{k} E_{t}\left\{\widehat{m c}_{t+k}\right\}
$$

así, $\widehat{m c}_{t} \equiv m c_{t}-m c$ es la (log) desviación de costo marginal real de su valor de steady-state $m c=-\log \left(\frac{\varepsilon}{\varepsilon-1}\right) \equiv-\mu$.

Se puede reescribir la expresión anterior en una forma más compacta como

$$
\bar{P}_{H, t}-P_{H, t-1}=\beta \theta E_{t}\left\{\bar{p}_{H, t+1}-p_{H, t}\right\}+\pi_{H, t}+(1-\beta \theta) \widehat{m c}_{t}
$$

Alternativamente, usando la relación $\widehat{m c}_{t}=m c_{t}^{n}-p_{H, t}+\mu$ para substituir $\widehat{m c}_{t}$ en la ecuación anterior,y después de realizar algunas manipulaciones, obtenemos la regla de fijación de precios en términos de los costos marginales nominales esperados:

$$
\bar{P}_{H, t}=\mu+(1-\beta \theta) \sum_{k=0}^{\infty}(\beta \theta)^{k} E_{t}\left\{\widehat{m c}_{t+k}^{n}\right\}
$$

Bajo el supuesto de fijación de precios, la dinámica del índice de precios doméstico se representa mediante la siguiente ecuación

$$
P_{H, t} \equiv\left[\theta P_{H, t-1}^{1-\varepsilon}+(1-\theta) \bar{p}_{H, t}^{1-\varepsilon}\right]^{\frac{1}{1-\varepsilon}}
$$

la cual puede ser log-linealizada en su steady-state para obtener

$$
\pi_{H, t}=(1-\theta)\left(\bar{p}_{H, t}-p_{H, t-1}\right) .
$$

Finalmente, resulta posible combinar la expresión anterior con la ecuación (A.4) para obtener

$$
\pi_{H, t}=\beta E_{t}\left\{\pi_{H, t+1}\right\}+\lambda \widehat{m c}_{t}
$$

donde $\lambda \equiv \frac{(1-\theta)(1-\beta \theta)}{\theta}$.

\section{A.2. Maximización de Utilidad}

\section{A.2.1. Agente $h$}

Para cada agente de tipo $h$, el problema de maximización está dado por:

$$
\mathscr{L}_{\left\{c_{h, t}, n_{h, t}\right\}}=E_{0} \sum_{t=0}^{\infty} \beta^{t}\left[\frac{c_{h, t}^{1-\sigma}}{1-\sigma}-\frac{n_{h, t}^{1-\varphi}}{1-\varphi}-\lambda_{t}\left(P_{h, t}(i) c_{h, t}(i)+E\left\{Q_{h, t+1} D_{h, t+1}\right\}-D_{h, t}-W_{t} \tilde{\ell} k_{h, t}-T_{h, t}\right)\right.
$$

y las condiciones de primer orden vienen dadas por: 


$$
\begin{gathered}
\frac{\partial \mathscr{L}}{\partial c_{h, t}}: c_{h, t}^{-\sigma}-\lambda_{t} P_{t}=0 \\
\lambda_{t}=\frac{c_{h, t}^{-\sigma}}{P_{t}} \\
\frac{\partial \mathscr{L}}{\partial n_{h, t}}:-n_{h, t}^{\varphi}+\lambda_{t} W_{t}=0 \\
\lambda_{t}=\frac{n_{h, t}^{\varphi}}{W_{t}} \\
\Rightarrow \frac{1}{c_{h, t}^{\sigma} P_{t}}=\frac{n_{h, t}^{\varphi}}{W_{t}} \Rightarrow \frac{1}{c_{h, t}^{\sigma} P_{t}}=\frac{\left(\tilde{\ell} k_{h, t}\right)^{\varphi}}{W_{t}} \\
\frac{\partial \mathscr{L}}{\partial D_{h, t+1}}: \lambda_{t} E_{t}\left\{Q_{h, t, t+1}\right\}=0 \\
\lambda_{t+1}=0
\end{gathered}
$$

lo cual puede expresarse como:

$$
\begin{aligned}
& \beta E_{t}\left(\lambda_{t+1}\right)=E_{t}\left(Q_{h, t, t+1}\right) \lambda_{t} \\
& \beta E_{t}\left(\frac{\lambda_{t+1}}{\lambda_{t}}\right)=E_{t}\left(Q_{h, t, t+1}\right) \\
& \beta E_{t}\left[\frac{\left(c_{h, t+1}\right)^{-\sigma}}{P_{t+1}} \frac{P_{t}}{\left(c_{h, t}\right)^{-\sigma}}\right]=E_{t}\left(Q_{h, t, t+1}\right)
\end{aligned}
$$

en el que si se ordenan los términos tenemos la ecuación de

$$
\beta R_{t} E_{t}\left\{\left(\frac{c_{h, t+1}}{c_{h, t}}\right)^{-\sigma}\left(\frac{P_{t}}{P_{t+1}}\right)\right\}=1
$$

donde $R_{t}^{-1}=E_{t}\left\{Q_{h, t, t+1}\right\}$ representa el precio de un bono de 1 período libre de riesgo (denominado en moneda local), y por lo tanto $R_{t}$ representa el retorno neto.

\section{A.2.2. Análisis}

Dado las ecuaciones (A.8) y (A.9), tomando logaritmo a ambos lados:

1.

$$
\begin{aligned}
& \overline{p c} \widehat{p}_{h, t}+\overline{p c} \widehat{p}_{h, t}+\bar{D} \widehat{D}_{h, t}-\overline{D R} D_{h, t}-\overline{D R} \widehat{R}_{t}-\bar{W} \tilde{\ell} \widehat{k} \widehat{W}_{t}-\bar{W} \tilde{\ell} \bar{k} \widehat{k}_{t} \\
& \text { por cada agente } h
\end{aligned}
$$


2.

$$
\begin{aligned}
& \beta R_{t} E_{t}\left\{\left(\frac{c_{h, t+1}}{c_{h, t}}\right)^{-\sigma}\left(\frac{P_{t}}{P_{t+1}}\right)\right\}=1 \\
& \log \beta+\log R_{t}-\log c_{h, t}^{-\sigma}+\log E_{t}\left(c_{h, t+1}\right)^{-\sigma}+\log E_{t}\left(\frac{P_{t}}{P_{t+1}}\right)=\log (1) \\
& \widehat{c}_{h, t}=E_{t}\left[\widehat{c}_{h, t+1}\right]-\frac{1}{\sigma}\left[-\rho+r-E_{t}\left\{\pi_{t+1}\right\}\right]
\end{aligned}
$$

donde la ultima ecuación surge de la relación $\pi_{t+1}=\log P_{t+1}-\log P_{t}$.

\section{A.2.3. Log-Linealización}

1.

$$
\begin{aligned}
& \bar{C}_{t} \mathrm{e}^{C_{t}}=\vartheta \bar{C}_{t}^{*} \overline{\mathcal{Q}}_{t}^{\frac{1}{\sigma}} \mathrm{e}^{C_{t}^{*}+\mathcal{Q}_{t}^{\frac{1}{\sigma}}} \\
& \bar{C}_{t}\left(1+C_{t}\right)=\vartheta \bar{C}_{t}^{*} \overline{\mathcal{Q}}_{t}^{\frac{1}{\sigma}}\left(1+C_{t}^{*}+\mathcal{Q}_{t}^{\frac{1}{\sigma}}\right) \\
& \bar{C}_{t} C_{t}=\bar{C}_{t}^{*} \overline{\mathcal{Q}}_{t}^{\frac{1}{\sigma}}\left(C_{t}^{*}+\mathcal{Q}_{t}^{\frac{1}{\sigma}}\right) \\
& c_{t}=c_{t}^{*}+\frac{1}{\sigma} q_{t} \\
& c_{t}=c_{t}^{*}+\left(\frac{1-\alpha}{\sigma}\right) s_{t}
\end{aligned}
$$

2 .

$$
\begin{aligned}
\log \left(\frac{M C_{t}^{n}}{\bar{M} C^{n}}\right) & =\log \left(\frac{(1-\tau)\left(\frac{W_{t}}{A_{t}}\right)\left(P_{H, t}\right)}{(1-\tau)\left(\frac{\bar{W}}{A}\right)\left(\bar{P}_{H}\right)}\right) \\
m a_{t} & =-\nu+w_{t}-a_{t}-p_{H, t}
\end{aligned}
$$

\section{A.3. Costos Marginales}

1. Tomamos (3.31) y reemplazando por (3.19), (3.12), $y_{t}^{*}=c_{t}^{*},(3.11)$ y (3.23), obtenemos:

$$
\begin{aligned}
m c_{t} & =-\nu+p_{t}+\sigma\left(y_{t}^{*}+\left(\frac{(1-\alpha)}{\sigma}\right) s_{t}\right)+\varphi\left(y_{t}-a_{t}\right)-a_{t}-p_{H, t} \\
& =-\nu+p_{t}-p_{H, t}+\sigma y_{t}^{*}+q_{t}+\varphi\left(y_{t}-a_{t}\right)-a_{t} \\
& =-\nu+\left(y_{t}-y_{t}^{*}\right) \frac{\sigma}{\omega_{\alpha}}+\sigma y_{t}^{*}+\varphi\left(y_{t}-a_{t}\right)-a_{t}
\end{aligned}
$$

2. Con las ecuaciones (3.32) y (3.19) tenemos:

$$
\begin{aligned}
m a_{t}^{*} & =-\nu^{*}+p_{t}^{*}+\sigma y_{t}^{*} \varphi\left(y_{t}^{*}-a_{t}^{*}\right)-a_{t}^{*}-p_{t}^{*} \\
& =-\nu^{*}+\sigma y_{t}^{*} \varphi\left(y_{t}^{*}-a_{t}^{*}\right)-a_{t}^{*}
\end{aligned}
$$


A partir de las ecuaciones (3.33), (3.34) y $m c_{t}=m c^{*}=-\mu$

1.

$$
\begin{aligned}
& -\mu=-\nu+\left(\frac{\sigma}{\omega_{\alpha}}+\varphi\right) y_{t}+\sigma\left(1-\frac{1}{\omega_{\alpha}}\right) y_{t}^{*}-(1+\varphi) a_{t} \\
& -\mu+\nu-\sigma\left(1-\frac{1}{\omega_{\alpha}}\right) y_{t}^{*}+(1+\varphi) a_{t}=\left(\frac{\sigma}{\omega_{\alpha}}+\varphi\right) y_{t} \\
& y_{t}=\frac{\omega_{\alpha}(\nu-\mu)}{\sigma+\varphi \omega_{\alpha}}+\frac{\sigma\left(1-\omega_{\alpha}\right)}{\sigma+\varphi \omega_{\alpha}} y_{t}^{*}+\frac{\omega_{\alpha}(1+\varphi)}{\sigma+\varphi \omega_{\alpha}} a_{t}
\end{aligned}
$$

2.

$$
\begin{aligned}
& m c_{t}^{*}=-\nu^{*}+(\sigma+\varphi) y_{t}^{*}-(1+\varphi) a_{t}^{*} \\
& -\mu+\nu^{*}+(1+\varphi) a_{t}^{*}=(\sigma+\varphi) y_{t}^{*} \\
& y_{t}^{*}=\frac{\nu^{*}-\mu}{\sigma+\varphi}+\frac{1+\varphi}{\sigma+\varphi} a_{t}^{*}
\end{aligned}
$$

De las ecuaciones (3.33) y (3.35) tenemos

$$
\begin{aligned}
& y_{t}^{*}=\frac{\widehat{m c_{t}}-\mu+\nu-\left(\frac{\sigma}{\omega_{\alpha}}+\varphi\right) y_{t}+(1+\varphi) a_{t}}{\sigma\left(1-\frac{1}{\omega_{\alpha}}\right)} \\
& \text { and } y_{t}^{*}=\frac{\bar{y}-\frac{\omega_{\alpha}(\nu-\mu)}{\sigma+\varphi \omega_{\alpha}}-\frac{\omega_{\alpha}(1+\varphi)}{\sigma+\varphi \omega_{\alpha}} a_{t}}{\frac{\sigma\left(1-\omega_{\alpha}\right)}{\sigma+\varphi \omega_{\alpha}}}
\end{aligned}
$$

por lo tanto

$$
\begin{aligned}
& \frac{\widehat{m c}_{t}-\mu+\nu-\left(\frac{\sigma}{\omega_{\alpha}}+\varphi\right) y_{t}+(1+\varphi) a_{t}}{\sigma\left(1-\frac{1}{\omega_{\alpha}}\right)}=\frac{\bar{y}-\frac{\omega_{\alpha}(\nu-\mu)}{\sigma+\varphi \omega_{\alpha}}-\frac{\omega_{\alpha}(1+\varphi)}{\sigma+\varphi \omega_{\alpha}} a_{t}}{\frac{\sigma\left(1-\omega_{\alpha}\right)}{\sigma+\varphi \omega_{\alpha}}} \\
& \Rightarrow\left(\frac{-1}{-1}\right)\left[\frac{\left.\omega_{\alpha} \widehat{m c}_{t}+\omega_{\alpha}(\nu-\mu)-\omega_{\alpha}\left(\frac{\sigma}{\omega_{\alpha}}+\varphi\right) y_{t}+\omega_{\alpha}(1+\varphi) a_{t}\right]}{\left(\omega_{\alpha}-1\right)}\right]= \\
& =\frac{\bar{y}}{\left(1-\omega_{\alpha}\right)}-\frac{\omega_{\alpha}(\nu-\mu)}{\left(1-\omega_{\alpha}\right)}-\frac{\omega_{\alpha}(1+\varphi) a_{t}}{\left(1-\omega_{\alpha}\right)} \\
& \Rightarrow \frac{-\omega_{\alpha} \widehat{m c} \omega_{t}}{\left(1-\omega_{\alpha}\right)}-\frac{\omega_{\alpha}(\nu-\mu)}{\left(1-\omega_{\alpha}\right)}+\frac{\omega_{\alpha}\left(\frac{\sigma}{\omega_{\alpha}}+\varphi\right) y_{t}}{\left(1-\omega_{\alpha}\right)}-\frac{\omega_{\alpha}(1+\varphi) a_{t}}{\left(1-\omega_{\alpha}\right)}= \\
& =\frac{\bar{y}}{\left(1-\omega_{\alpha}\right)}\left(\sigma+\varphi \omega_{\alpha}\right)-\frac{\omega_{\alpha}(\nu-\mu)}{\left(1-\omega_{\alpha}\right)}-\frac{\omega_{\alpha}(1+\varphi) a_{t}}{\left(1-\omega_{\alpha}\right)}
\end{aligned}
$$


Por lo que obtenemos un expresión simplificada de $\widehat{m c}_{t}$ en relación con $y_{t}$ y $\bar{y}$ :

$$
\widehat{m c}_{t}=\left(\frac{\sigma}{\omega_{\alpha}}+\varphi\right) y_{t}-\left(\frac{\sigma}{\omega_{\alpha}}+\varphi\right) \bar{y}
$$

Para los costos marginales de la economía mundial procedemos de la siguiente manera, primero, expresamos (3.34) y (3.36) en términos de $a_{t}^{*}$ :

$$
\begin{aligned}
& a_{t}^{*}=\frac{-\left[m c_{t}^{*}+\nu^{*}-(\sigma+\varphi) y_{t}^{*}\right]}{(1+\varphi)}=\frac{\bar{y}^{*}-\frac{\nu^{*}-\mu}{\sigma+\varphi}}{\frac{1+\varphi}{\sigma+\varphi}} \\
& \frac{-\widehat{m c}_{t}^{*}}{(1+\varphi)}-\frac{\nu^{*}-\mu}{(1+\varphi)}+\frac{(\sigma+\varphi)}{(1+\varphi)} y_{t}^{*}=\frac{(\sigma+\varphi)}{(1+\varphi)} \bar{y}^{*}-\frac{\nu^{*}-\mu}{(1+\varphi)}
\end{aligned}
$$

y obtenemos los costos marginales de la economía mundial en relación con $y_{t}^{*}$ y $\bar{y}^{*}$ :

$$
\widehat{m c}_{t}^{*}=(\sigma+\varphi) y_{t}^{*}-(\sigma+\varphi) \bar{y}^{*}
$$

\section{A.3.1. Derivación de las ecuaciones IS}

Para la economía mundial utilizamos 3.24 y la relación $\tilde{y}_{t}^{*}=y_{t}^{*}-\bar{y}_{t}^{*}$ a efectos de obtener:

$$
\begin{aligned}
& \tilde{y}_{t}^{*}+\bar{y}_{t}=E_{t}\left\{\tilde{y}_{t+1}^{*}+\bar{y}_{t+1}^{*}\right\}-\frac{1}{\sigma}\left(r_{t}^{*}-E_{t}\left\{\pi_{t+1}^{*}\right\}-\rho\right) \\
& \tilde{y}_{t}^{*}=E_{t}\left\{\tilde{y}_{t+1}^{*}\right\}-\frac{1}{\sigma}\left(r_{t}^{*}-E_{t}\left\{\pi_{t+1}^{*}\right\}-\rho\right)+E_{t}\left\{\bar{y}_{t+1}^{*}\right\}-\bar{y}_{t}^{*}
\end{aligned}
$$

las ultimas dos expresiones pueden expresarse como (teniendo $a_{t}^{*}=\rho_{\alpha} a_{t-1}^{*}+\epsilon_{t}^{*}$, and $\Omega_{0}+\Gamma_{0} a_{t}^{*}$ ):

$$
\begin{aligned}
E_{t}\left\{\bar{y}_{t+1}^{*}\right\}-\bar{y}_{t}^{*} & =E_{t}\left\{\Omega_{0}+\Gamma_{0} a_{t+1}^{*}\right\}-\Omega_{0}+\Gamma_{0} a_{t}^{*} \\
& =\Omega_{0}+\Gamma_{0} E_{t}\left\{a_{t+1}^{*}\right\}-\Omega_{0}-\Gamma_{0} a_{t}^{*} \\
& =\Gamma_{0}\left[E_{t}\left\{a_{t+1}^{*}\right\}-a_{t}^{*}\right] \\
& =\Gamma_{0}[\rho_{\alpha}^{*} a_{t}^{*}-\underbrace{E_{t}\left\{\epsilon_{t+1}^{*}\right\}}_{=0}-a_{t}^{*}]
\end{aligned}
$$

y obtenemos la ecuación dinámica IS de la economía mundial:

$$
\tilde{y}_{t}^{*}=E_{t}\left\{\tilde{y}_{t+1}^{*}\right\}-\frac{1}{\sigma}\left(r_{t}^{*}-E_{t}\left\{\pi_{t+1}^{*}\right\}-\rho\right)+\Gamma_{0} a_{t}^{*}\left(\rho_{\alpha}^{*}-1\right)
$$

Del mismo modo, resulta fácil obtener las mismas relaciones para la economía abierta:

$$
\tilde{y}_{t}=E_{t}\left\{\tilde{y}_{t+1}\right\}-\frac{\omega_{\alpha}}{\sigma}\left(r_{t}-E_{t}\left\{\pi_{H, t+1}\right\}-\bar{r}_{t}\right) \frac{\omega_{\alpha}(1+\varphi)\left(1-\rho_{\alpha}\right)}{\sigma+\omega_{\alpha \varphi}} a_{t}-\varphi \Theta_{\alpha} E_{t}\left\{\Delta y_{t+1}^{*}\right\}
$$




\section{A.3.2. Objetivo $C P I$}

Dado $p_{H, t}=-\alpha s_{t}$, si se lo sustituye para $s_{t}$ en 3.26, insertando la ecuación resultante en 3.33, obtenemos

$$
\begin{aligned}
& m c_{t}=-\nu+\frac{\sigma}{\omega_{\alpha}}-\frac{P_{H, t}}{\alpha}+\varphi y_{t}^{*}-\varphi \frac{\omega_{\alpha}}{\sigma} \frac{P_{H, t}}{\alpha}+\sigma y_{t}^{*}-\frac{\sigma}{\omega_{\alpha}} y_{t}^{*}-(1-\varphi) a_{t} \\
& m c_{t}=-\frac{1}{\alpha}\left(1+\frac{\varphi \omega_{\alpha}}{\sigma}\right) p_{H, t}-(1+\varphi)\left(a_{t}-a_{t}^{*}\right)
\end{aligned}
$$

Introduciendo la ultima expresión en 3.26, y combinándola con las ecuaciones 3.33 y 3.29, resulta:

$$
\gamma_{c} p_{H, t}=p_{H, t-1}+\beta E_{t}\left\{p_{H, t+1}\right\}-\lambda(1+\varphi)\left(a_{t}-a_{t}^{*}\right)
$$




\section{Apéndice B}

\section{Solución de la Dinámica mediante el método de los coeficientes indeterminados}

\section{B.1. Revisión del Toolkit}

En esta sección, se presenta el sistema de ecuaciones a efectos de presentar una intuición de como se resuelve utilizando la solución propuesta por Uhlig [7]. Dado $x_{t}$, de dimensión $m \times 1$, el vector de variables de estado endógenos, $y_{t}$, de dimensión $n \times 1$ las variables de control endógenos y $z_{t}$, que se nota el proceso estocástico endógeno de dimensión $k \times 1$. LA relación de equilibrio de estas variables viene dado por

$$
\begin{aligned}
& 0=A x_{t}+B x_{t-1}+C y_{t}+D z_{t} \\
& 0=E_{t}\left[F x_{t+1}+G x_{t}+H x_{t-1}+J y_{t+1}+K y_{t}+L z_{t+1}+M z_{t}\right] \\
& z_{t+1}=N z_{t}+\varepsilon_{t+1} ; \quad E_{t}\left[\varepsilon_{t+1}\right]=0
\end{aligned}
$$

donde se supone que:

- $C$ is of size $l \times n, l \geq n$ and of rank $n$,

- $F$ is of size $(m+n-l) \times n$,

- and $N$ has only stable eigenvalues.

al final de esta sección se presentan las matrices obtenidas tras el proceso de log-linealización a efectos de implementarlos en el Toolkit.

EL propósito de este sistema es el de encontrar la función de reacción recursiva:

$$
\begin{aligned}
x_{t} & =P x_{t-1}+Q z_{t} \\
y_{t} & =R x_{t-1}+S z_{t} \\
z_{t+1} & =N z_{t}
\end{aligned}
$$

Para resolver este problema se necesita resolver la matriz de ecuación cuadrática, lo cual se demostrará en la siguiente sección. La solución de equilibrio se caracteriza con el siguiente teorema 
Teorema B.1.1. Dado la Función de reacción recursiva que resuelve las ecuaciones (B.4), (B.5) y (B.6), entonces la matriz de coeficientes se puede obtener de la siguiente manera. Dado $C^{+}$la matriz pseudo inversa ${ }^{4}$ de $C, y C^{0}$ la matriz nula de $C$.

1. P satisface la matriz de ecuaciones cuadrática

$$
\begin{aligned}
& 0=C^{0} A P+C^{0} B \\
& 0=\left(F-J C^{+} A\right) P^{2}-\left(J C^{+} B-G+K C^{+} A\right) P-K C^{+} B+H
\end{aligned}
$$

2. $R$ satisface

$$
R=-C^{+}(A P+B)
$$

3. Dado P y R, siendo $V$ la matriz

$$
V=\left[\begin{array}{cc}
I_{k} \otimes A, & I_{k} \otimes C \\
N^{\prime} \otimes F+I_{k} \otimes(F P+J R+G), & N^{\prime} \otimes J+I_{k} \otimes K
\end{array}\right]
$$

donde $I_{k}$ es la matriz identidad de dimensión $k \times k$, entonces se puede obtener $Q$ y $S$ mediante:

$$
V\left[\begin{array}{c}
\operatorname{vec}(Q) \\
\operatorname{vec}(S)
\end{array}\right]=-\left[\begin{array}{c}
\operatorname{vec}(D) \\
\operatorname{vec}(L N+M)
\end{array}\right]
$$

donde la función vec(.) denota la vectorización.

Prueba. Insertando la función de reacción en la ecuación (B.1). Resulta

$$
0=(A P+C R+B) x_{t-1}+(A Q+C S+D) z_{t}
$$

que se debe cumplir para $x_{t-1} y z_{t}$. Así, las matrices de coeficientes $x_{t-1} y z_{t}$ in(B.10) son cero. Insertando dos veces la función de reacción en la ecuación (B.2) y utilizando (B.3) obtenemos:

$$
\begin{aligned}
0= & ((F P+J R+G) P+K R+H) x_{t-1}+ \\
& ((F Q+J S+L) N+(F P+J R+G) Q+K S+M) z_{t}
\end{aligned}
$$

Lo que nuevamente las matrices de coeficientes $x_{t-1} y z_{t}$ necesitan ser. Vectorizando la matriz de coeficientes $z_{t}$ en las ecuaciones (B.10) y (B.11) y uniendo términos en vec $(Q)$ y vec $(S)$ resulta la fórmula para $Q$ y $S$.

El próximo paso consiste en encontrar la solución para $P$ y $R$, por esta razón, reescribimos la matriz de coeficientes $x_{t-1}$ en la ecuación (B.10) como:

$$
\begin{aligned}
R & =-C^{+}(A P+B) \\
0 & =C^{0} A P+C^{0} B
\end{aligned}
$$

notar que la matriz $\left[\left(C^{+}\right),\left(C^{0}\right)^{\prime}\right]$ es no-singular y que $C^{0} C=0$. Si empleamos (B.12) para reemplazar $R$ en la matriz de coeficientes $x_{t-1}$ en (B.11), obtenemos (B.8).

\footnotetext{
${ }^{4}$ Ya que $C$ es de rango completo, resulta: $C^{+}=\left(C^{\prime} C\right)^{-1} \cdot C^{\prime}$
} 


\section{B.1.1. Solución de la Matriz de Ecuación Cuadrática}

En esta sección resolvemos las Matrices de Ecuaciones Cuadráticas (B.7) y (B.8). La Matriz $P$ satisface:

$$
\Psi P^{2}-\Gamma P-\Theta=0
$$

mediante las ecuaciones (B.7) y (B.8), definimos

$$
\begin{aligned}
\Psi & =\left[\begin{array}{c}
0_{l-n, m} \\
F-J C^{+} A
\end{array}\right] \\
\Gamma & =\left[\begin{array}{c}
C^{0} A \\
J C^{+} B-G+K C^{+} A
\end{array}\right] \\
\Theta & =\left[\begin{array}{c}
C^{0} B \\
K C^{+} B-H
\end{array}\right]
\end{aligned}
$$

donde $0_{l-n, m}$ es una matriz $(l-n) \times m$ con todos los valores igual a cero. La ecuación (B.13) puede resolverse mediante un problema de autovalores y autovectores. Dado el autovalor $\lambda$ y el autovector $s$ de la matriz $\Xi$ con respecto a la matriz $\Delta$ que constituyen un vector y un valor que satisfacen:

$$
\lambda \Delta s=\Xi s
$$

Teorema B.1.2. Para resolver la matriz de ecuación cuadrática (B.13) para la matriz $P$ de $m \times m$, dado $\Gamma$ y $\Theta$ de $m \times m$, definimos las matrices $\Xi$ y $\Delta$ de $2 m \times 2 m$ como:

$$
\begin{aligned}
& \Xi=\left[\begin{array}{cc}
\Gamma & \Theta \\
I_{m} & 0_{m, m}
\end{array}\right] \\
& \Delta=\left[\begin{array}{cc}
\Psi & 0_{m, m} \\
0_{m, m} & I_{m}
\end{array}\right]
\end{aligned}
$$

donde $I_{m}$ es la matriz identidad de dimensión $m, y 0_{m, m}$ es la matriz $m \times m$ con todos sus valores igual a cero.

1. Si s es un autovector y $\lambda$ el autovalor de $\Xi$ con respecto a $\Delta$, s puede reescribirse como $s^{\prime}=\left[\lambda x^{\prime}, x^{\prime}\right]$ para algún $x \in \mathbb{R}^{m}$.

2. Si existen $m$ autovalores $\lambda_{1}, \ldots, \lambda_{m}$ con los respectivos autovectores $s_{1}, \ldots, s_{m}$ de $\Xi$ con respecto a $\Delta$, escrito como $s_{i}^{\prime}=\left[\lambda_{i} x_{i}^{\prime}, x_{i}^{\prime}\right]$ para algún $x_{i} \in \mathbb{R}^{m}, y$ si $\left(x_{1}, \ldots, x_{m}\right)$ es linealmente independiente, entonces

$$
P=\Omega \Lambda \Omega^{-1}
$$

es la solución para la matriz de ecuación cuadrática (B.13), donde $\Omega=\left[x_{1}, \ldots, x_{m}\right]$ y $\Lambda=\operatorname{diag}\left(\lambda_{1}, \ldots, \lambda_{m}\right)$. 
Prueba. En primer lugar, examinamos las ultimas $m$ filas de la ecuación (B.14) y observamos que cualquier autovector s por algún autovalor $\lambda$ de la matriz $\Xi$ con respecto a $\Delta$ puede expresarse como:

$$
s=\left[\begin{array}{c}
\lambda x \\
x
\end{array}\right]
$$

para algún $x \in \mathbb{R}^{m}$ dado la forma de $\Xi y \Delta$. Examinando las primeras $m$ filas de la ecuación (B.14) entonces observamos que:

$$
\lambda^{2} \Psi x-\lambda \Gamma x-\Theta x=0
$$

A continuación

$$
\Psi \Omega \Lambda^{2}-\Gamma \Omega \Lambda-\Theta \Omega=0
$$

y por lo tanto

$$
\Psi P^{2}-\Gamma P-\Theta=0
$$

luego multiplicando con $\Omega^{-1}$ a la derecha. Invirtiendo los pasos surge que la solución diagonalizable de P para (B.13) se puede escribir de esta forma.

\section{B.1.2. Implementado las ecuaciones en el Toolkit OID}

En esta sección se plantean las ecuaciones que se necesitan para implementar el Toolkit, la cual, puede ampliarse a cualquier número de consumidores.

1. de (3.41): $r_{t}^{*}=\rho-\sigma\left(1-\rho_{a}^{*}\right) \Gamma_{0} a_{t}^{*}$

2. el CPI: $\pi_{t}=p_{t}-p_{t-1}$

3. CPI en DIT (3.44): $e_{t}=\frac{\sigma(1+\varphi)}{\sigma+\omega_{\alpha \varphi}} a_{t}-\frac{\sigma(1+\varphi)}{\sigma+\omega_{\alpha \varphi}} a_{t}^{*}$

4. $(3.43) q_{t}=e_{t}(1-\alpha)$

5. $c_{t}=\Phi_{\alpha} y_{t}+\left(1-\Phi_{\alpha}\right) y_{t}^{*}$

6. condicion de equilibrio: $c^{*}=y^{*}$

7. de (3.35): $y_{t}=\Gamma_{\alpha} a_{t}+\Theta_{\alpha} y_{t}^{*}$

8. de $(3.36): y_{t}^{*}=\Gamma_{0} a_{t}^{*}$

9. de (3.11): $s_{t}=\frac{1}{\alpha} p_{t}-\frac{1}{\alpha} p_{H, t}$

10. $r^{C P I}=r_{t}-\pi_{t}$

11. $-c_{t}+\sum_{h} c_{h}=0$

12. $-D_{t}+\sum_{h} D_{h}=0$ 
13. $-N_{t}+\sum_{h} n_{h}=0$

14. $\overline{p c} \widehat{p}_{h, t}+\overline{p c} \widehat{c}_{h, t}+\bar{D} \widehat{D}_{h, t}-\overline{D R} D_{h, t}-\overline{D R} \widehat{R}_{t}-\bar{W} \widetilde{\ell} \widehat{k} \widehat{W}_{t}-\bar{W} \widetilde{\ell} \widehat{k} \widehat{k}_{t}$

15. $k_{h}-z_{t}-g_{h}=0$

16. de (3.38): $\pi_{t}^{*}=\beta E_{t}\left\{\pi_{t+1}^{*}\right\}+\kappa_{0} \tilde{y}_{t}^{*}$

17. de (3.40): $\tilde{y}_{t}^{*}=E_{t}\left\{\tilde{y}_{t+1}^{*}\right\}-\frac{1}{\sigma}\left(r_{t}^{*}-E_{t}\left\{\pi_{t+1}^{*}\right\}-\overline{r r}_{t}^{*}\right)$

18. de (3.37): $\pi_{H, t}=\beta E_{t}\left\{\pi_{H, t+1}\right\}+\kappa_{\alpha} \tilde{y}_{t}$

19. de (3.39): $\tilde{y}_{t}=E_{t}\left\{\tilde{y}_{t+1}\right\}-\frac{\omega_{\alpha}}{\sigma}\left(r_{t}-E_{t}\left\{\pi_{H, t+1}\right\}-\overline{r r}_{t}\right)$

20. de (3.42): $r_{t}=\bar{r} r_{t}$

21. de (3.44): $p_{t}=\frac{\alpha \sigma(1+\varphi)}{\sigma+\omega_{\alpha \varphi}} a_{t}-\frac{\alpha \sigma(1+\varphi)}{\sigma+\omega_{\alpha \varphi}} a_{t}^{*}$

22. $p_{H, t}=0$

23. de (3.28): $n x_{t}=\frac{\alpha \Lambda}{\omega_{\alpha}} y_{t}-\frac{\alpha \Lambda}{\omega_{\alpha}} y_{t}^{*}$

24. $\widehat{c}_{h, t}=E_{t}\left[\widehat{c}_{h, t+1}\right]-\frac{1}{\sigma}\left[-\rho+r-E_{t}\left\{\pi_{t+1}\right\}\right]$ 